\title{
EFFECT OF UV RADIATION ON THE SPECTRAL FINGERPRINTS OF EARTH-LIKE PLANETS ORBITING M STARS
}

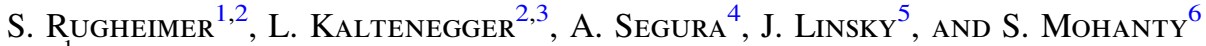 \\ ${ }^{1}$ Harvard Smithsonian Center for Astrophysics, 60 Garden Street, Cambridge, MA 02138, USA \\ ${ }^{2}$ Carl Sagan Institute, Cornell University, Ithaca, NY 14853, USA \\ ${ }^{3}$ MPIA, Koenigstuhl 17, D-69117 Heidelberg, Germany \\ ${ }^{4}$ Instituto de Ciencias Nucleares, Universidad Nacional Autónoma de México, México \\ 5 JILA, University of Colorado and NIST, 440 UCB, Boulder, CO 80309-0440, USA \\ ${ }^{6}$ Imperial College London, 1010 Blackett Lab, Prince Consort Road, London SW7 2AZ, UK \\ Received 2014 August 18; accepted 2015 June 10; published 2015 August 10
}

\begin{abstract}
We model the atmospheres and spectra of Earth-like planets orbiting the entire grid of $\mathrm{M}$ dwarfs for active and inactive stellar models with $T_{\text {eff }}=2300 \mathrm{~K}$ to $T_{\text {eff }}=3800 \mathrm{~K}$ and for six observed MUSCLES M dwarfs with UV radiation data. We set the Earth-like planets at the $1 \mathrm{AU}$ equivalent distance and show spectra from the visible to IR $(0.4-20 \mu \mathrm{m})$ to compare detectability of features in different wavelength ranges with the James Webb Space Telescope and other future ground- and spaced-based missions to characterize exo-Earths. We focus on the effect of UV activity levels on detectable atmospheric features that indicate habitability on Earth, namely, $\mathrm{H}_{2} \mathrm{O}, \mathrm{O}_{3}, \mathrm{CH}_{4}$, $\mathrm{N}_{2} \mathrm{O}$, and $\mathrm{CH}_{3} \mathrm{Cl}$. To observe signatures of life $-\mathrm{O}_{2} / \mathrm{O}_{3}$ in combination with reducing species like $\mathrm{CH}_{4}-$ we find that early and active $\mathrm{M}$ dwarfs are the best targets of the $\mathrm{M}$ star grid for future telescopes. The $\mathrm{O}_{2}$ spectral feature at $0.76 \mu \mathrm{m}$ is increasingly difficult to detect in reflected light of later $\mathrm{M}$ dwarfs owing to low stellar flux in that wavelength region. $\mathrm{N}_{2} \mathrm{O}$, another biosignature detectable in the IR, builds up to observable concentrations in our planetary models around $\mathrm{M}$ dwarfs with low UV flux. $\mathrm{CH}_{3} \mathrm{Cl}$ could become detectable, depending on the depth of the overlapping $\mathrm{N}_{2} \mathrm{O}$ feature. We present a spectral database of Earth-like planets around cool stars for directly imaged planets as a framework for interpreting future light curves, direct imaging, and secondary eclipse measurements of the atmospheres of terrestrial planets in the habitable zone to design and assess future telescope capabilities.
\end{abstract}

Key words: astrobiology - planets and satellites: atmospheres - planets and satellites: terrestrial planets - stars: low-mass

\section{INTRODUCTION}

About 2000 extrasolar planets have been found to date, with thousands more awaiting confirmation from space- and groundbased searches. Several of these planets have been found in or near the circumstellar habitable zone (see, e.g., Udry et al. 2007; Borucki et al. 2011, 2013; Kaltenegger \& Sasselov 2011; Batalha et al. 2013; Kaltenegger et al. 2013; Quintana et al. 2014), with masses and radii consistent with rocky planet models. Future mission concepts to characterize Earth-like planets are designed to take spectra of extrasolar planets with the ultimate goal of remotely detecting atmospheric signatures (e.g., Beichman et al. 1999, 2006; Cash 2006; Kaltenegger et al. 2006; Traub et al. 2006). Several proposed missions are designed to characterize nearby super-Earth and Earth-like planets using emergent visible and infrared spectra. For transiting terrestrial planets, the James Web Space Telescope (JWST; see Gardner et al. 2006; Deming et al. 2009; Kaltenegger \& Traub 2009), as well as future ground- and space-based telescopes (Snellen et al. 2013; Rodler and LópezMorales 2014), will search for biosignatures in a rocky planet's atmosphere. NASA's explorer mission Transiting Exoplanet Survey Satellite (TESS) is designed to search the whole sky for potentially habitable planets around the closest and brightest stars to Earth (et al. 2014), for eventual follow-up with JWST and large ground-based observatories such as the E-ELT or GMT.

In our solar neighborhood, $75 \%$ of stars are $\mathrm{M}$ dwarfs. The abundance of M dwarfs, as well as the contrast ratio and transit probability, favors the detection of planets in the habitable zone of $\mathrm{M}$ dwarfs. Therefore, it is likely that the first habitable planet suitable for follow-up observations will be found orbiting a nearby M dwarf (Dressing \& Charbonneau 2013). The M spectral class is very diverse, spanning nearly three orders of magnitude in luminosity and an order of magnitude in mass.

The UV environment of a host star dominates the photochemistry and therefore the resulting atmospheric constituents, including biosignatures for terrestrial planets (see, e.g., Segura et al. 2005; Hu et al. 2012; Rugheimer et al. 2013; Domagal-Goldman et al. 2014; Grenfell et al. 2014; Tian et al. 2014). To date, few observations exist in the UV region for $M$ dwarfs. Previously, only UV spectra of very active flare stars, such as $\mathrm{AD}$ Leo, were available from the IUE satellite, primarily during flares and for a few quiescent phases. The MUSCLES program observed chromospheric emission from six weakly active M dwarfs with Hubble Space Telescope (HST; France et al. 2013). Note that Ly $\alpha$ is by far the strongest line in the UV for M dwarfs. Since the core of the intrinsic stellar Ly $\alpha$ emission is absorbed by neutral hydrogen in the interstellar medium, one must reconstruct the line to compensate for this absorption to get accurate flux levels (see, e.g., Wood et al. 2005; Linsky et al. 2013).

Several groups have explored the effect of a different stellar spectral type on the atmospheric composition of Earth-like planets by primarily considering one star, AD Leo, as a template for the diverse range of $\mathrm{M}$ dwarfs, and by using the extreme limit of inactivity, photosphere-only, PHOENIX models (Segura et al. 2005; Kitzmann et al. 2011a, 2011b; 
Wordsworth et al. 2011; Grenfell et al. 2014). In this paper we expand on this work by establishing planetary atmosphere models for the full $\mathrm{M}$ dwarf main sequence, using a stellar temperature grid from 3800 to $2400 \mathrm{~K}$, including recent $H S T$ observations of six $\mathrm{M}$ dwarfs to explore the effect of different spectral energy distributions (SEDs) on terrestrial atmosphere models and on detectable atmospheric signatures, including biosignatures. Atmospheric biosignatures are remotely detectable chemical species in the atmosphere that are by-products of life processes. Recent research shows that possible false positives may occur under certain geological settings (Domagal-Goldman et al. 2014; Tian et al. 2014; Wordsworth \& Pierrehumbert 2014).

We create a grid of $M$ dwarf input spectra, which can be used to probe the entire range of UV flux levels. To showcase the whole range, we simulate model planets around host stars at the two extreme limits of activity: active and inactive stellar models. We compare these models to the six observed $\mathrm{M}$ dwarfs with recent UV observations (France et al. 2013).

We explore the influence of stellar UV flux on the atmospheric structure, chemical abundance, and spectral features for Earth-like planets, including the observability of biosignatures in the visible to IR. We focus our analysis on spectral biosignatures for a temperate rocky planet like Earth, $\mathrm{O}_{3}, \mathrm{O}_{2}, \mathrm{CH}_{4}, \mathrm{~N}_{2} \mathrm{O}$, and $\mathrm{CH}_{3} \mathrm{Cl}$, and those that indicate habitability, $\mathrm{H}_{2} \mathrm{O}$ and $\mathrm{CO}_{2}$ (Lovelock 1975; Sagan et al. 1993; Des Marais et al. 2002).

In Section 2, we describe our model, and Section 3 presents the influence of stellar types on the abundance of atmospheric chemical species. In Section 4, we examine the remote observability of such spectral features, and in Sections 5 and 6 , we summarize the results and discuss their implications.

\section{MODEL DESCRIPTION}

\subsection{Stellar M Dwarf Spectral Grid Model}

M dwarfs span nearly three orders of magnitude in luminosity and remain active for much longer timescales than earlier stellar types. As main-sequence stars age, their UV flux levels decrease even as their bolometric luminosity increases. Understanding the UV flux incident on a planetary atmosphere is critical to understanding and interpreting future observations of atmospheric constituents, including biosignatures.

Current stellar models are unable to model the UV region from $M$ dwarfs self-consistently for three main reasons. First, complex magnetic fields responsible for heating the chromosphere are thought to drive much of the UV activity and have thus far been ignored in stellar models. Second, the models are missing opacities in the UV. Third, the models are semiempirical, with no energy conservation to balance magnetic heating with radiative losses. These problems are being addressed by current work, and models will be available in the future to test against M dwarf UV observations.

We generate stellar input models for the entire $M$ dwarf spectral class (M0 to M9) to explore the boundaries of the UV environment of an exoplanet orbiting a main-sequence star. We create two sets of models based on the extreme limits of stellar activity. For the purposes of this paper, "active" stellar models are constructed to represent the most active $M$ dwarf measurements, and "inactive" semi-empirical models without chromospheres are constructed to represent the lowest theoretical UV flux field. We compare these limiting-case

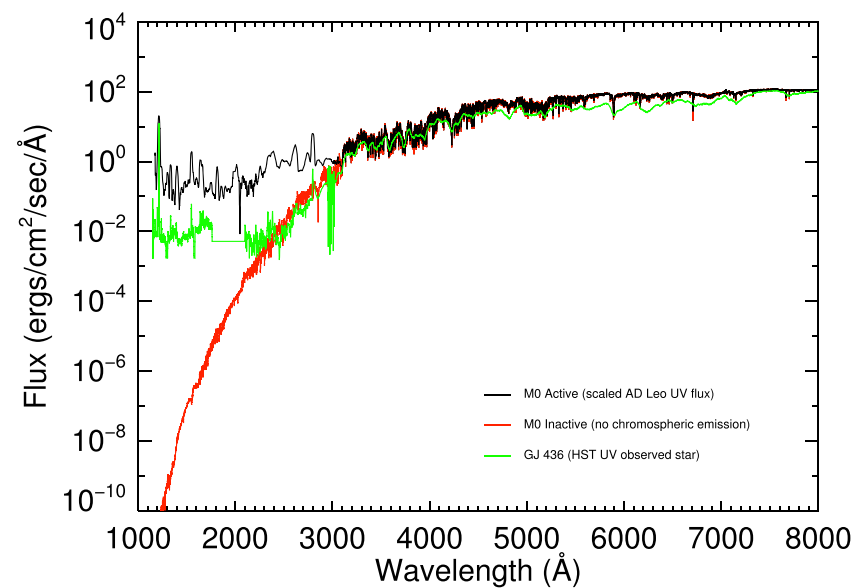

Figure 1. Stellar input spectra at the top of the atmosphere (TOA) of Earthlike planets at the 1 AU equivalent of an M0 "active" star with UV flux scaled from AD Leo (black), "inactive" PHOENIX model of M0, and HST UV observations plus photosphere model for GJ 436 (green).

models with six well-observed $\mathrm{M}$ dwarfs that show significant chromospheric flux (see Figure 1) despite being traditionally classified as quiescent stars owing to the presence of $\mathrm{H} \alpha$ in absorption (France et al. 2013). A few M dwarfs observed with GALEX in the near-ultraviolet (near-UV; 1750-2750 $\AA$ ) show near-photospheric continuum level fluxes (as computed with PHOENIX models) and can be classified as inactive even though the exact UV flux level has not been measured yet. For example, Gl 445 and Gl 682 have roughly $90 \%$ of the observed NUV flux density predicted by PHOENIX model photospheres (Shkolnik \& Barman 2014). More observations are needed to determine the ultimate lower limit of UV flux emitted by old and/or late $\mathrm{M}$ dwarfs. To compare our calculations for inactive star models with published work, we use theoretical photosphere PHOENIX models as a lower bound (Allard 2014).

The chromospheric far-ultraviolet (far-UV) flux from $\mathrm{M}$, $\mathrm{L}$, and $\mathrm{T}$ dwarfs (i.e., very low mass stars and brown dwarfs), while crucial for determining the potential habitability of any planets around them, is very poorly characterized through direct observations. Given their common chromospheric origin, $\mathrm{H} \alpha$, $\mathrm{Ly} \alpha, \mathrm{Ca}$ II $\mathrm{H}$ and $\mathrm{K}$, and $\mathrm{Mg}$ II $\mathrm{H}$ and $\mathrm{K}$ emission lines have all been used as proxies for FUV activity. However, many of these lines are inaccessible to $\mathrm{M}$ dwarfs because they are at shorter wavelengths, where these stars are intrinsically less luminous. For M dwarfs, $\mathrm{H} \alpha$ emission, in particular, has been studied extensively and to date has the most robust data set for each $\mathrm{M}$ dwarf spectral type compared with the other lines considered above (West et al. 2004, 2011). In addition, Jones \& West (2015) shows that $\mathrm{H} \alpha$ fluxes correlate with NUV fluxes from GALEX. Therefore, we use $\mathrm{H} \alpha$ to estimate the FUV emission for our active stellar grid by scaling the known FUV and $\mathrm{H} \alpha$ emission from the active M3.5 star AD Leo (see Equation (1) and Figure 2). Very active, early M dwarfs (M0M5) are known to saturate in the $\mathrm{H} \alpha$ emission around $\log$ $\left(L_{\mathrm{H}_{\alpha}} / L_{\text {bol }}\right)=-3.75$ (Hawley et al. 1996; West et al. 2004; Reiners \& Basri 2008).

West et al. (2004) tabulate the $\log \left(\right.$ mean $\left.L_{\mathrm{H}_{\alpha}} / L_{\text {bol }}\right)$ versus spectral type from M0 to L0 from observations of 1910 active M dwarfs. We parameterize the data from West et al. (2004) to derive a relationship of UV to mean $\mathrm{H} \alpha$ emission for each spectral subtype in the $M$ dwarf class (see Figure 2 and 


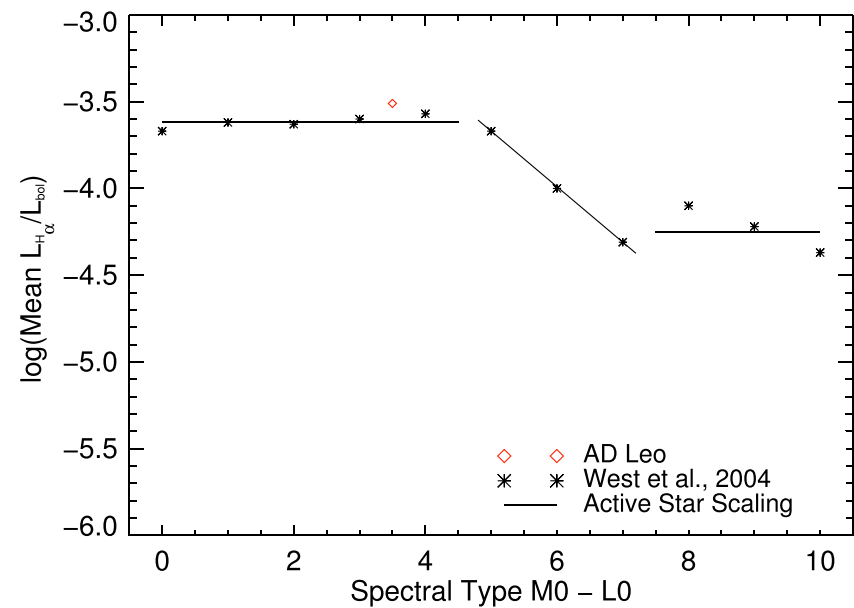

Figure 2. Scaling used for active stellar models (black line) overplotted on West et al. (2004) measurements of $\log$ (mean $L_{\mathrm{H}_{\alpha}} / L_{\text {bol }}$ ) (black asterisks).

Equation (1)):

$$
\begin{aligned}
\log \left(L_{\mathrm{H}_{\alpha} *} / L_{\text {bol } *}\right) & =-3.62 \text { for M0-M4 } \\
\log \left(L_{\mathrm{H}_{\alpha} *} / L_{\text {bol } *}\right) & =-0.32 \times(M)-2.07 \\
\text { where } M & =5,6,7 \text { for M5, M6, M7 } \\
\log \left(\mathrm{L}_{\mathrm{H}_{\alpha} *} / \mathrm{L}_{\text {bol } *}\right) & =-4.25 \text { for M8-M9. }
\end{aligned}
$$

We use the $\mathrm{H} \alpha / \mathrm{UV}$ scaling of AD Leo, which has both a wellcharacterized UV spectrum and $\mathrm{H} \alpha$ emission measurement as a calibration data point to set the scale. AD Leo, an M3.5 eV star, has $\log \left(L_{\mathrm{H}_{\alpha}} / L_{\text {bol }}\right)=-3.51$ (Walkowicz \& Hawley 2009) and has been well observed in the UV (see Segura et al. 2005 for AD Leo IUE spectrum and Linsky et al. 2013 for reconstructed Ly $\alpha$ flux). The M dwarf active spectrum is then given by

$$
\begin{aligned}
F_{\mathrm{FUV} *}= & F_{\mathrm{FUV} \text { AD Leo }} \times\left(\frac{T_{\text {eff } *}}{T_{\text {eff AD Leo }}}\right)^{4} \\
& \times \frac{\log \left(L_{\mathrm{H}_{\alpha} *} / L_{\text {bol } *}\right)}{\log \left(L_{\mathrm{H}_{\alpha} \text { ADLeo }} / L_{\text {bolADLeo }}\right)} .
\end{aligned}
$$

Our stellar temperature grid (Figure 3) covers the full $\mathrm{M}$ dwarf spectral range, with the stellar parameters for active and inactive models given in Table 1 . For the active star models, we scale the UV flux in the wavelength range 1000-3000 $\AA$ from AD Leo's observed UV spectrum to the other spectral types according to Equation (2). For each active model star on our grid we concatenated a solar-metallicity, unreddened synthetic PHOENIX spectrum, which only considers photospheric emission (Allard 2014), from 3000 to $45450 \AA$ to the scaled observations from the IUE archive for AD Leo from 1000 to $3000 \AA$ A combined with the reconstructed Ly $\alpha$ from Linsky et al. (2013) for the region 1210-1222 $\AA^{7}{ }^{7}$ Note that young active stars are highly variable, especially in the UV. AD Leo is the most active and well-characterized $\mathrm{M}$ dwarf. Our active models therefore represent the extreme high end of activity for each stellar type from an active flare star. For comparison, the

\footnotetext{
http://archive.stsci.edu/iue/
}

inactive semi-empirical models include no chromospheric emission. The continuum originating from the photosphere is taken from a PHOENIX model with the same stellar parameters as the corresponding active star from 1000-45450 ̊. Thus, these models provide only a lower limit to the stellar UV flux. More observations are needed to determine the true lower limit of inactive $\mathrm{M}$ dwarfs in the UV.

The models represent the extreme limits of the UV radiation environment for an exoplanet using a flare star for the upper bound and a photosphere-only model for the lower bound in the UV. Figure 1 shows that the UV flux region between those two limits spans over 10 orders of magnitude in the FUV. We use the observations of six $M$ dwarfs from the MUSCLES program to probe the region in between our active and inactive models. The MUSCLES stars have been traditionally classified as "quiescent" because they do not present $\mathrm{H} \alpha$ in emission.

The six M dwarfs were observed by two UV spectrographs (COS and STIS) on HST (France et al. 2013). We joined these HST measurements ${ }^{8}$ with the star's corresponding PHOENIX models at $2800 \AA$ after adjusting the HST flux levels to the level a planet would receive at the $1 \mathrm{AU}$ equivalent in the habitable zone. The input parameters for the PHOENIX models are the observed star's $T_{\text {eff }},[\mathrm{Fe} / \mathrm{H}], \log g$, and the rotation velocity, $v \sin i$, as summarized in Table 2 and described for each star based on the most recent observations in the Appendix. When observations of $v \sin i$ were not available, we used instead the peak of the observed distribution for 56 M dwarfs (Jenkins et al. 2009).

The MUSCLES database interpolates the region between 1760 and $2100 \AA$ because the noise in the observations was large in that region since those wavelengths were covered by the lowest-sensitivity part of the STIS G230L bandpass. In order to not bias the results too high or too low, the MUSCLES team used the average flux in the 2100-2200 $\AA$ region to interpolate the 1760-2100 A region. Follow-up observations to the MUSCLES program with a new HST Treasury program aim to probe the $1760-2100 \AA$ region of the spectrum with higher sensitivity. We used the MUSCLES database and interpolation as given. Because this region of the UV is important for $\mathrm{O}_{3}$ production, uncertainty of this interpolation could influence our atmospheric results.

Input active stellar spectra and MUSCLES spectra are shown in Figure 3 (inactive models are not shown).

\subsection{Planetary Atmosphere Model}

We use EXO-P (Kaltenegger \& Sasselov 2010), a coupled $1 \mathrm{D}$ radiative-convective atmosphere code developed for rocky exoplanets. The code incorporates a 1D climate (Kasting \& Ackerman 1986; Pavlov et al. 2000; Haqq-Misra et al. 2008), 1D photochemistry (Pavlov \& Kasting 2002; Segura et al. 2005, 2007) and 1D radiative transfer model (Traub \& Stier 1976; Kaltenegger \& Traub 2009) to calculate the model spectrum of an Earth-like exoplanet orbiting host $\mathbf{M}$ dwarfs in the habitable zone.

EXO-P is a model that simulates both the effects of stellar radiation on a planetary environment and the planet's outgoing spectrum. We model an altitude range that extends upward to $60 \mathrm{~km}$ with 100 height layers. We use a geometrical model in which the average 1D global atmospheric model profile is

\footnotetext{
http://cos.colorado.edu/ kevinf/muscles.html
} 

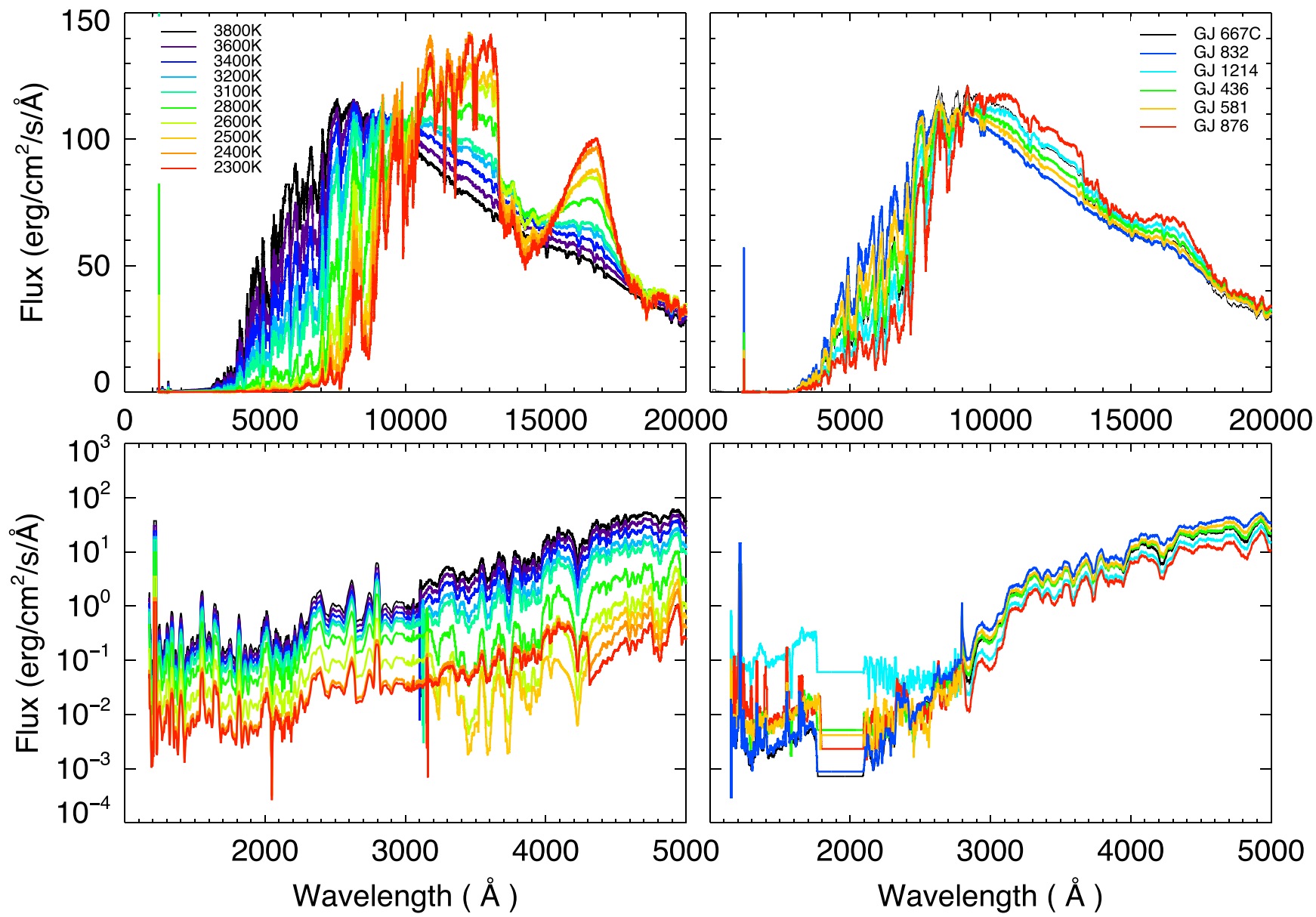

Figure 3. Top: stellar input spectra from 1000 to 20000 Å for the M0-M9 active grid stars with UV scaled by $\mathrm{H}_{\alpha}$ and AD Leo UV flux (left) and MUSCLES stars with HST UV observations (right). Bottom: UV input stellar fluxes in log scale.

Table 1

Stellar Properties for Active and Inactive Models

\begin{tabular}{lccc}
\hline \hline Star & $T_{\text {eff }}(\mathrm{K})$ & Mass $\left(M_{\odot}\right)$ & Radius $\left(R_{\odot}\right)$ \\
\hline M0 & 3800 & 0.60 & 0.62 \\
M1 & 3600 & 0.49 & 0.49 \\
M2 & 3400 & 0.44 & 0.44 \\
M3 & 3200 & 0.36 & 0.39 \\
M4 & 3100 & 0.2 & 0.36 \\
M5 & 2800 & 0.14 & 0.2 \\
M6 & 2600 & 0.10 & 0.15 \\
M7 & 2500 & 0.09 & 0.12 \\
M8 & 2400 & 0.08 & 0.11 \\
M9 & 2300 & 0.075 & 0.08 \\
\hline
\end{tabular}

generated using a plane-parallel atmosphere, treating the planet as a Lambertian sphere, and setting the stellar zenith angle to $60^{\circ}$ to represent the average incoming stellar flux on the dayside of the planet (see also Schindler \& Kasting 2000). The temperature in each layer is calculated from the difference between the incoming and outgoing flux and the heat capacity of the atmosphere in each layer. If the lapse rate of a given layer is larger than the adiabatic lapse rate, it is adjusted to the adiabatic rate until the atmosphere reaches equilibrium. We use a two-stream approximation (see Toon et al. 1989), which includes multiple scattering by atmospheric gases, in the visible/near-IR (NIR) to calculate the short-wave fluxes. Fourterm, correlated- $k$ coefficients parameterize the absorption by
$\mathrm{O}_{3}, \mathrm{H}_{2} \mathrm{O}, \mathrm{O}_{2}$, and $\mathrm{CH}_{4}$ (Pavlov et al. 2000). In the thermal IR region, a rapid radiative transfer model calculates the longwave fluxes. Clouds are not explicitly calculated. The effects of clouds on the temperature versus pressure profile are included by adjusting the surface albedo of the Earth-Sun system to have a surface temperature of $288 \mathrm{~K}$ (see Kasting et al. 1984; Pavlov et al. 2000; Segura et al. 2003, 2005). The photochemistry code, originally developed by Kasting et al. (1985), solves for 55 chemical species linked by 220 reactions using a reverse-Euler method (see Segura et al. 2010, and references therein). The photochemical model is stationary, and convergence is achieved when the following criteria are fulfilled: the production and loss rates of chemical species are balanced, which results in a steady state for the chemical concentrations, and the initial boundary conditions, such as surface mixing ratios or surface fluxes, are met.

The radiative transfer model used to compute planetary spectra is based on a model originally developed for trace gas retrieval in Earth's atmospheric spectra (Traub \& Stier 1976) and further developed for exoplanet transmission and emergent spectra (Kaltenegger et al. 2007, 2013; Kaltenegger \& Traub 2009; Kaltenegger 2010; Kaltenegger \& Sasselov 2010). In this paper, we model Earth's reflected and thermal emission spectra using 21 of the most spectroscopically significant molecules $\left(\mathrm{H}_{2} \mathrm{O}, \mathrm{O}_{3}, \mathrm{O}_{2}, \mathrm{CH}_{4}, \mathrm{CO}_{2}, \mathrm{OH}, \mathrm{CH}_{3} \mathrm{Cl}, \mathrm{NO}_{2}, \mathrm{~N}_{2} \mathrm{O}\right.$, $\mathrm{HNO}_{3}, \mathrm{CO}, \mathrm{H}_{2} \mathrm{~S}, \mathrm{SO}_{2}, \mathrm{H}_{2} \mathrm{O}_{2}, \mathrm{NO}, \mathrm{ClO}, \mathrm{HOCl}, \mathrm{HO}_{2}, \mathrm{H}_{2} \mathrm{CO}$, $\mathrm{N}_{2} \mathrm{O}_{5}$, and $\mathrm{HCl}$ ). We use a Lambert sphere as an approximation for the disk-integrated planet in our model. The surface of our 
Table 2

Stellar Properties for MUSCLES Model Stellar Spectra

\begin{tabular}{|c|c|c|c|c|c|c|}
\hline $\begin{array}{l}\text { MUSCLES } \\
\text { Stars }\end{array}$ & $T_{\text {eff }}(\mathrm{K})$ & Radius $\left(R_{\odot}\right)$ & {$[\mathrm{Fe} / \mathrm{H}]$} & $\log g$ & Age (Gyr) & $\begin{array}{c}v \sin i \\
\left(\mathrm{~km} \mathrm{~s}^{-1}\right)\end{array}$ \\
\hline GJ 832 & 3620 & 0.48 & -0.12 & 4.70 & $\ldots$ & 3 \\
\hline GJ 1214 & 3250 & 0.211 & +0.05 & 4.99 & $6 \pm 3$ & $<1$ \\
\hline GJ 436 & 3416 & 0.455 & +0.04 & 4.83 & $6.5-9.9$ & $<1$ \\
\hline GJ 581 & 3498 & 0.299 & -0.10 & 4.96 & $7-11$ & 2.1 \\
\hline GJ 876 & 3129 & 0.3761 & +0.19 & 4.89 & $0.1-5$ & 1.38 \\
\hline
\end{tabular}

Note. For GJ 832 and GJ 667C there is no $v \sin i$ measurement. We assumed a $v \sin i=3.0$ corresponding to the peak of the distribution (Jenkins et al. 2009) when generating a PHOENIX model. See text for references.

model planet corresponds to Earth's current surface of $70 \%$ ocean, $2 \%$ coast, and $28 \%$ land. The land surface consists of $30 \%$ grass, $30 \%$ trees, $9 \%$ granite, $9 \%$ basalt, $15 \%$ snow, and $7 \%$ sand. Surface reflectivities are taken from the USGS Digital Spectral Library ${ }^{9}$ and the ASTER Spectral Library ${ }^{10}$ (following Kaltenegger et al. 2007).

Clouds have a strong impact on the detectability of atmospheric species. For the spectra shown in Figures 7-9, we assume a $60 \%$ global cloud cover with cloud layers analogous to Earth (40\% water clouds at $1 \mathrm{~km}, 40 \%$ water clouds at $6 \mathrm{~km}$, and $20 \%$ ice clouds at $12 \mathrm{~km}$, following Kaltenegger et al. 2007). In the visible to NIR, clouds increase the reflectivity of an Earth-like planet substantially and therefore increase the equivalent widths of all observable features, even though clouds block access to some of the lower atmosphere. In the IR, clouds slightly decrease the overall emitted flux of an Earth-like planet because they radiate at lower temperatures and therefore decrease the equivalent widths of all observable absorption features, even though they can increase the relative depth of a spectral feature as a result of lowering the continuum temperature of the planet. For a comparison of scenarios with Earth-analog clouds to those of clear sky spectra see Rugheimer et al. (2013).

Using 34 layers, we calculate the spectrum at high spectral resolution with several points per line width. The line shapes and widths are computed using Doppler and pressure broadening on a line-by-line basis for each layer in the model atmosphere. The overall high-resolution spectrum is calculated with $0.1 \mathrm{~cm}^{-1}$ wavenumber steps. The figures are shown smoothed to a resolving power of 150 in the IR and 800 in the visible using a triangular smoothing kernel. The spectra may be binned further for comparison with proposed future spectroscopy missions designed to characterize Earth-like planets. We previously validated EXO-P from the visible to the IR using data from ground and space (Kaltenegger et al. 2007; Kaltenegger \& Traub 2009; Rugheimer et al. 2013).

\subsection{Simulation Setup}

To examine the effects of the varying UV flux of M dwarfs on an Earth-like atmosphere and its observable spectral features, we use the temperature grid of stellar models ranging from M9 to M0 ( $\left.T_{\text {eff }}=3800-2300 \mathrm{~K}\right)$ for active and inactive models, along with the six $\mathrm{M}$ dwarfs with well-characterized UV fluxes from HST (see Section 2.2). We simulated an Earthlike planet with the same mass as Earth at the $1 \mathrm{AU}$ equivalent

\footnotetext{
9 http://speclab.cr.usgs.gov/spectral-lib.html

${ }^{10} \mathrm{http} / / /$ speclib.jpl.nasa.gov
}

orbital distance, defined by the wavelength integrated stellar flux received on top of the planet's atmosphere being equivalent to $1 \mathrm{AU}$ in our solar system, calculated by $1 \mathrm{AU}_{\text {eq }}=\mathrm{AU} \times \sqrt{\left(R / R_{\odot}\right)^{2} \times\left(T_{\text {eff }} / T_{\text {eff } \odot}\right)^{4}}$.

The biogenic (produced by living organisms) fluxes were held fixed in the models in accordance with the fluxes that reproduce the modern mixing ratios in the Earth-Sun case, except for the cooler $\mathrm{M}$ dwarfs, where $\mathrm{CH}_{4}$ and $\mathrm{N}_{2} \mathrm{O}$ were given a fixed mixing ratio of $1.0 \times 10^{-3}$ and $1.5 \times 10^{-2}$, respectively (following Segura et al. 2003, 2005). The surface fluxes for the long-lived gases $\mathrm{H}_{2}, \mathrm{CH}_{4}, \mathrm{~N}_{2} \mathrm{O}, \mathrm{CO}$, and $\mathrm{CH}_{3} \mathrm{Cl}$ were calculated such that the Earth around the Sun yields a $T_{\text {surf }}=288 \mathrm{~K}$ for surface mixing ratios: $\mathrm{cH}_{2}=5.5 \times 10^{-7}$, $\mathrm{cCH}_{4}=1.6 \times 10^{-6}, \quad \mathrm{cCO}_{2}=3.5 \times 10^{-4}, \quad \mathrm{cN}_{2} \mathrm{O}=$ $3.0 \times 10^{-7}, \mathrm{cCO}=9.0 \times 10^{-8}$, and $\mathrm{cCH}_{3} \mathrm{Cl}=5.0 \times 10^{-10}$ (see Rugheimer et al. 2013). The corresponding input surface fluxes to the atmosphere are $-1.9 \times 10^{12} \mathrm{~g} \mathrm{H}_{2} \mathrm{yr}^{-1}$, $5.3 \times 10^{14} \mathrm{~g} \mathrm{CH}_{4} \mathrm{yr}^{-1}, 7.9 \times 10^{12} \mathrm{~g} \mathrm{~N}_{2} \mathrm{O} \mathrm{yr}^{-1}, 1.8 \times 10^{15} \mathrm{~g}$ $\mathrm{CO} \mathrm{yr}-1$, and $4.3 \times 10^{12} \mathrm{~g} \mathrm{CH}_{3} \mathrm{Cl} \mathrm{yr}^{-1}$. The $\mathrm{N}_{2}$ mixing ratio is set to be a "fill gas" such that the total surface pressure is 1 bar. These boundary conditions were used for M0-M5 active grid stars, M0-M3 inactive grid stars, and all MUSCLES stars.

For M6-M9 active grid stars and for M4-M9 inactive grid stars, the boundary condition for $\mathrm{CH}_{4}$ is changed to a fixed mixing ratio once the UV environment of the host star drops below a certain level because $\mathrm{CO}, \mathrm{H}_{2}$, and $\mathrm{CH}_{4}$ do not converge according to the criteria described in Section 2.2 assuming a modern Earth biological flux (see also Segura et al. 2005). In reality, the thermodynamical cost for microbes producing $\mathrm{CH}_{4}$ would become unprofitable as temperatures would initially rise owing to the increased greenhouse effect, causing the microbes to operate less efficiently. This type of biological feedback is not included in the models. $\mathrm{CH}_{4}$ is produced biotically by methanogens and other organisms and abiotically through hydrothermal vent systems. In the modern atmosphere there is a significant anthropogenic source of $\mathrm{CH}_{4}$ from natural gas, livestock, and rice paddies. Upper limits of abiotic fluxes of methane can be estimated for terrestrial planets following Guzmán-Marmolejo et al. (2014). An estimate of Earth biotic methane is about 30 times the abiotic flux (see discussion in Segura et al. 2005), and thus a range of methane fluxes may be maintained on a terrestrial planet via methanogenesis in addition to abiotic methane production.

Therefore, we set the mixing ratio of $\mathrm{CH}_{4}$ to $1 \times 10^{-3}$, corresponding to a value of the last stable run with an Earthlike biological $\mathrm{CH}_{4}$ flux for an active M5 star for planets 
around host stars that show this "runaway" behavior. Given these boundary conditions, the methane flux necessary to sustain a $1 \times 10^{-3}$ mixing ratio of methane is $5.65 \times 10^{14}$ $\mathrm{g} \mathrm{yr}^{-1}$ (equal to our present Earth methane flux) for the planet around the hottest $\mathrm{M}$ dwarf $\left(T_{\text {eff }}=3800 \mathrm{~K}\right)$, either active or inactive, $1.16 \times 10^{14} \mathrm{~g} \mathrm{yr}^{-1}(20.5 \%$ of the Earth value) for the planet around the coolest $\left(T_{\text {eff }}=2300 \mathrm{~K}\right)$ active star, and $5.87 \times 10^{12} \mathrm{~g} \mathrm{yr}^{-1}$ (1\% of the Earth value) for the coolest inactive $\mathrm{M}$ dwarf of our sample.

For the less active stars, we set the deposition velocity (rate at which they are deposited to the surface) for $\mathrm{H}_{2}$ and $\mathrm{CO}$ to $2.4 \times 10^{-4} \mathrm{~cm} \mathrm{~s}^{-1}$ and $1.2 \times 10^{-4} \mathrm{~cm} \mathrm{~s}^{-1}$, respectively (see also Kharecha et al. 2005; Segura et al. 2005). Rauer et al. (2011) used a deposition velocity for $\mathrm{H}_{2}$ of $7.7 \times 10^{-4} \mathrm{~cm} \mathrm{~s}^{-1}$. This number was obtained after calibrating the model to reproduce the $\mathrm{H}_{2}$ atmospheric abundance measured for present Earth. In our case, the deposition values for $\mathrm{H}_{2}$ and $\mathrm{CO}$ correspond to maximum air-sea transfer rates estimated using the "piston velocity" approach (Broecker 1982; Kharecha et al. 2005). Since a larger $\mathrm{H}_{2}$ deposition velocity implies consumption by bacteria, we use the abiological value in Kharecha et al. (2005).

We followed a similar procedure for constraining the $\mathrm{N}_{2} \mathrm{O}$ concentrations for M6-M9 inactive stars. We set the mixing ratio of $\mathrm{N}_{2} \mathrm{O}$ to $1.5 \times 10^{-2}$, corresponding to values of the last stable run with Earth-like biological fluxes, to prevent unphysical buildup of $\mathrm{N}_{2} \mathrm{O} . \mathrm{N}_{2} \mathrm{O}$ is emitted primarily by (de) nitrifying bacteria with anthropogenic sources from fertilizers in agriculture, biomass burning, industry, and livestock and is a relatively minor constituent of the modern atmosphere at around 320 parts per billion (ppb), compared to the preindustrial concentration of $270 \mathrm{ppb}$ (Forster et al. 2007).

We used modern Earth fluxes for all of the MUSCLES stars without any "runaway" buildup of reduced gases. The $\mathrm{CH}_{4}$ and $\mathrm{N}_{2} \mathrm{O}$ fluxes are given in Table 3.

All of our simulations used a fixed mixing ratio of $355 \mathrm{ppm}$ for $\mathrm{CO}_{2}, 21 \% \mathrm{O}_{2}$, and a fixed upper boundary of $10^{-4}$ bar $(\sim 60 \mathrm{~km})$. In the 1D climate model, a surface albedo of 0.2 is fixed for all simulations, corresponding to the surface albedo that reproduces Earth's average temperature of $288 \mathrm{~K}$ for the Earth/Sun case. The planetary Bond albedo (surface + atmosphere) is calculated by the 1D climate code.

\section{ATMOSPHERIC MODEL RESULTS}

The amount of UV radiation emitted from the host star influences the abundances of major chemical atmospheric constituents, including $\mathrm{H}_{2} \mathrm{O}, \mathrm{CH}_{4}$, and $\mathrm{O}_{3}$, and, as a result, modifies the temperature-pressure profile of a planet. The UV fluxes incident at the top of the atmosphere of an Earth-like planet in the habitable zone are given in Table 4 and shown in Figure 3. An M0 active star model has 6.7 times more total UV flux than an M0 inactive star model. An M9 active star model has 4600 times more total UV flux than an M9 inactive star model. The greatest differences are in the FUV (1000-2000 А), where an active star model has $6.8 \times 10^{4}$ and $1.3 \times 10^{11}$ more FUV flux than an inactive M0 and M9 model, respectively. The MUSCLES stars' UV environments fall between the active and inactive star models, consistent with their classification as being weakly active.

The temperature versus altitude profile and the $\mathrm{H}_{2} \mathrm{O}, \mathrm{O}_{3}$, $\mathrm{CH}_{4}$, and $\mathrm{N}_{2} \mathrm{O}$ mixing ratio profiles for all of the simulations are shown in Figure 4, with each row corresponding to active, inactive, and MUSCLES star models, respectively. $\mathrm{CH}_{3} \mathrm{Cl}$ profiles are not shown here, but follow the same trends as $\mathrm{CH}_{4}$. Since both $\mathrm{O}_{2}$ and $\mathrm{CO}_{2}$ are well mixed in the atmosphere, their vertical mixing ratio profiles of 0.21 and $355 \mathrm{ppm}$, respectively, are not shown.

In the first column of Figure 4, we show the changes in the temperature/altitude profile for Earth-like atmosphere models around $\mathrm{M}$ dwarfs for active (top row), inactive (middle row), and the six observed MUSCLES stars (bottom row). All temperature inversions are weaker than for the modern Earth because M dwarfs emit low UV flux in the 2000-3000 wavelength region, thereby producing near-isothermal stratospheres (see also Segura et al. 2005). Figure 4 shows that temperature inversions are weaker for the higher UV environment stars. This is counterintuitive since for the modern Earth, $\mathrm{O}_{3}$ absorption of $\mathrm{UV}$ radiation causes stratospheric heating and an inversion. However, in these lower UV environments, additional heating is provided by stratospheric $\mathrm{CH}_{4}$ and $\mathrm{H}_{2} \mathrm{O}$.

$\mathrm{H}_{2} \mathrm{O}$ concentrations are lower in the stratosphere for planets around $\mathrm{M}$ dwarf models with higher UV fluxes, although higher $\mathrm{O}_{3}$ concentrations will act as a shield, partially offsetting the effect of higher UV photon fluxes (Figure 4, second column). $\mathrm{H}_{2} \mathrm{O}$ can also be formed in the stratosphere from $\mathrm{CH}_{4}$ and $\mathrm{OH}\left(\mathrm{CH}_{4}+\mathrm{OH} \rightarrow \mathrm{CH}_{3}+\mathrm{H}_{2} \mathrm{O}\right)$ and by increased upward vertical transport in the nearly isothermal stratospheres of Earth-like planets orbiting $\mathrm{M}$ dwarfs (see also Segura et al. 2005). $\mathrm{O}_{3}$ shields $\mathrm{H}_{2} \mathrm{O}$ in the troposphere from UV environments. While photochemically inert in the troposphere, $\mathrm{H}_{2} \mathrm{O}$ can be removed by photolysis at wavelengths shortward of $2000 \AA$ in the stratosphere (see columns 2 and 3 in Table 4) or by reactions with excited oxygen, $\mathrm{O}\left({ }^{1} D\right)$, to produce $\mathrm{OH}$ radicals.

In an atmosphere containing $\mathrm{O}_{2}, \mathrm{O}_{3}$ concentrations are determined primarily by the absorption of UV light shortward of $2400 \AA$ in the stratosphere, and we see a corresponding decrease in $\mathrm{O}_{3}$ concentrations correlated with decreased FUV radiation for cooler $\mathrm{M}$ dwarfs, as well as inactive versus active stars (Figure 4, third column).

$\mathrm{CH}_{4}$ mixing ratio profiles are shown in column 4 of Figure 4. As mentioned previously, we set the mixing ratio of methane to be $1000 \mathrm{ppm}$ for the later $\mathrm{M}$ dwarf spectral types as described in Section 2.3 and Table 3. For Earth-like $\mathrm{CH}_{4}$ fluxes, $\mathrm{CH}_{4}$ concentrations decrease with higher UV environments owing to reactions with $\mathrm{OH}$ to form $\mathrm{H}_{2} \mathrm{O}$ and by photolysis by $\lambda<1500 \AA$ (Figure 4, fourth column).

$\mathrm{N}_{2} \mathrm{O}$ mixing ratios are larger for cooler $\mathrm{M}$ dwarfs because cooler stars have smaller UV fluxes at $\lambda<2200 \AA$ and thus lower photolysis rates. Around later $\mathrm{M}$ dwarfs, we see mixing ratios of $\mathrm{N}_{2} \mathrm{O}$ that increase unrealistically for the inactive stellar models M6-M9, similar to $\mathrm{CH}_{4}$ (Figure 4, fifth column). Since such a "runaway" effect should not be sustainable by biology, we cap the $\mathrm{N}_{2} \mathrm{O}$ to a fixed mixing ratio corresponding to the last stable run for the inactive model M5. $\mathrm{N}_{2} \mathrm{O}$ is also an indirect sink for stratospheric $\mathrm{O}_{3}$, since about $1 \%$ is converted to NO. Therefore, increasing $\mathrm{N}_{2} \mathrm{O}$ decreases $\mathrm{O}_{3}$ abundance.

$\mathrm{OH}$ concentrations decrease with decreasing UV levels (in cooler and inactive $\mathrm{M}$ dwarfs), and $\mathrm{OH}$ is a primary sink for many species in the atmosphere, including, but not limited to, many biologically interesting species such as $\mathrm{CH}_{4}, \mathrm{CH}_{3}, \mathrm{HCl}$, $\mathrm{H}_{2}, \mathrm{H}_{2} \mathrm{~S}$, and $\mathrm{CH}_{3} \mathrm{Cl}$. $\mathrm{CH}_{3} \mathrm{Cl}$ concentrations increase for cooler and inactive $\mathrm{M}$ dwarfs owing to decreased stellar UV flux. 
Table 3

$\mathrm{CH}_{4}$ and $\mathrm{N}_{2} \mathrm{O}$ Fluxes and Mixing Ratios and $\mathrm{O}_{3}$ Column Depths

\begin{tabular}{|c|c|c|c|c|c|c|c|}
\hline & $\begin{array}{c}\text { Surface } \\
\text { Mixing } \\
\text { Ratio } \mathrm{CH}_{4}\end{array}$ & $\begin{array}{c}\text { Flux }\left(\mathrm{g} \mathrm{yr}^{-1}\right) \\
\mathrm{CH}_{4}\end{array}$ & $\begin{array}{l}\% \text { Earth } \\
\mathrm{CH}_{4} \text { flux }\end{array}$ & $\begin{array}{c}\text { Surface } \\
\text { Mixing } \\
\text { Ratio } \mathrm{N}_{2} \mathrm{O}\end{array}$ & $\begin{array}{c}\text { Flux }\left(\mathrm{g} \mathrm{yr}^{-1}\right) \\
\mathrm{N}_{2} \mathrm{O}\end{array}$ & $\begin{array}{c}\% \text { Earth } \\
\mathrm{N}_{2} \mathrm{O} \text { flux }\end{array}$ & $\begin{array}{c}\mathrm{O}_{3} \text { Column } \\
\text { Depth } \\
\left(\mathrm{cm}^{-2}\right)\end{array}$ \\
\hline \multicolumn{8}{|c|}{ Active } \\
\hline M0 A & $330 \mathrm{ppm}$ & $5.65 \times 10^{14}$ & $100 \%$ & $0.70 \mathrm{ppm}$ & $7.99 \times 10^{12}$ & $100 \%$ & $4.1 \times 10^{18}$ \\
\hline M1 A & $370 \mathrm{ppm}$ & $5.65 \times 10^{14}$ & $100 \%$ & $0.70 \mathrm{ppm}$ & $7.99 \times 10^{12}$ & $100 \%$ & $4.0 \times 10^{18}$ \\
\hline M2 A & $450 \mathrm{ppm}$ & $5.65 \times 10^{14}$ & $100 \%$ & $0.76 \mathrm{ppm}$ & $7.99 \times 10^{12}$ & $100 \%$ & $3.8 \times 10^{18}$ \\
\hline M3 A & $570 \mathrm{ppm}$ & $5.65 \times 10^{14}$ & $100 \%$ & $0.82 \mathrm{ppm}$ & $7.99 \times 10^{12}$ & $100 \%$ & $3.6 \times 10^{18}$ \\
\hline M4 A & 910 ppm & $5.65 \times 10^{14}$ & $100 \%$ & $0.93 \mathrm{ppm}$ & $7.99 \times 10^{12}$ & $100 \%$ & $3.5 \times 10^{18}$ \\
\hline M5 A & $1000 \mathrm{ppm}$ & $5.65 \times 10^{14}$ & $100 \%$ & $0.98 \mathrm{ppm}$ & $7.99 \times 10^{12}$ & $100 \%$ & $2.6 \times 10^{18}$ \\
\hline M6 A & $1000 \mathrm{ppm}$ & $2.90 \times 10^{14}$ & $51.8 \%$ & 1.7 ppm & $7.99 \times 10^{12}$ & $100 \%$ & $3.1 \times 10^{18}$ \\
\hline M7 A & $1000 \mathrm{ppm}$ & $1.28 \times 10^{14}$ & $22.7 \%$ & $3.0 \mathrm{ppm}$ & $7.99 \times 10^{12}$ & $100 \%$ & $2.4 \times 10^{18}$ \\
\hline M8 A & $1000 \mathrm{ppm}$ & $1.36 \times 10^{14}$ & $24.0 \%$ & $3.1 \mathrm{ppm}$ & $7.99 \times 10^{12}$ & $100 \%$ & $2.5 \times 10^{18}$ \\
\hline M9 A & $1000 \mathrm{ppm}$ & $1.20 \times 10^{14}$ & $21.2 \%$ & $3.5 \mathrm{ppm}$ & $7.99 \times 10^{12}$ & $100 \%$ & $2.3 \times 10^{18}$ \\
\hline \multicolumn{8}{|c|}{ Inactive } \\
\hline M0 I & $490 \mathrm{ppm}$ & $5.65 \times 10^{14}$ & $100 \%$ & $34 \mathrm{ppm}$ & $7.99 \times 10^{12}$ & $100 \%$ & $1.7 \times 10^{18}$ \\
\hline M1 I & $580 \mathrm{ppm}$ & $5.65 \times 10^{14}$ & $100 \%$ & $64 \mathrm{ppm}$ & $7.99 \times 10^{12}$ & $100 \%$ & $1.5 \times 10^{18}$ \\
\hline M2 I & $650 \mathrm{ppm}$ & $5.65 \times 10^{14}$ & $100 \%$ & $120 \mathrm{ppm}$ & $7.99 \times 10^{12}$ & $100 \%$ & $1.4 \times 10^{18}$ \\
\hline M3 I & $1000 \mathrm{ppm}$ & $5.65 \times 10^{14}$ & $100 \%$ & $360 \mathrm{ppm}$ & $7.99 \times 10^{12}$ & $100 \%$ & $1.3 \times 10^{18}$ \\
\hline M4 I & $1000 \mathrm{ppm}$ & $4.15 \times 10^{14}$ & $73.5 \%$ & $670 \mathrm{ppm}$ & $7.99 \times 10^{12}$ & $100 \%$ & $1.0 \times 10^{18}$ \\
\hline M5 I & $1000 \mathrm{ppm}$ & $1.23 \times 10^{14}$ & $21.8 \%$ & $15000 \mathrm{ppm}$ & $7.99 \times 10^{12}$ & $100 \%$ & $4.5 \times 10^{17}$ \\
\hline M6 I & 1000 ppm & $2.42 \times 10^{13}$ & $4.3 \%$ & $15000 \mathrm{ppm}$ & $4.04 \times 10^{11}$ & $5.1 \%$ & $1.4 \times 10^{17}$ \\
\hline M7 I & $1000 \mathrm{ppm}$ & $1.67 \times 10^{13}$ & $3.0 \%$ & $15000 \mathrm{ppm}$ & $4.96 \times 10^{10}$ & $0.6 \%$ & $6.1 \times 10^{16}$ \\
\hline M8 I & $1000 \mathrm{ppm}$ & $1.34 \times 10^{13}$ & $2.4 \%$ & $15000 \mathrm{ppm}$ & $5.72 \times 10^{9}$ & $0.07 \%$ & $2.6 \times 10^{16}$ \\
\hline M9 I & $1000 \mathrm{ppm}$ & $5.87 \times 10^{12}$ & $1.0 \%$ & 15000 ppm & $3.17 \times 10^{9}$ & $0.04 \%$ & $1.1 \times 10^{16}$ \\
\hline \multicolumn{8}{|c|}{ MUSCLES } \\
\hline GJ 832 & $550 \mathrm{ppm}$ & $5.65 \times 10^{14}$ & $100 \%$ & $15 \mathrm{ppm}$ & $7.99 \times 10^{12}$ & $100 \%$ & $1.6 \times 10^{18}$ \\
\hline GJ $667 \mathrm{C}$ & $330 \mathrm{ppm}$ & $5.65 \times 10^{14}$ & $100 \%$ & $0.7 \mathrm{ppm}$ & $7.99 \times 10^{12}$ & $100 \%$ & $7.0 \times 10^{18}$ \\
\hline GJ 1214 & $1600 \mathrm{ppm}$ & $5.65 \times 10^{14}$ & $100 \%$ & $1.1 \mathrm{ppm}$ & $7.99 \times 10^{12}$ & $100 \%$ & $2.3 \times 10^{18}$ \\
\hline GJ 436 & $1600 \mathrm{ppm}$ & $5.65 \times 10^{14}$ & $100 \%$ & $4.3 \mathrm{ppm}$ & $7.99 \times 10^{12}$ & $100 \%$ & $1.1 \times 10^{18}$ \\
\hline GJ 581 & $1400 \mathrm{ppm}$ & $5.65 \times 10^{14}$ & $100 \%$ & $2.9 \mathrm{ppm}$ & $7.99 \times 10^{12}$ & $100 \%$ & $1.2 \times 10^{18}$ \\
\hline GJ 876 & $3400 \mathrm{ppm}$ & $5.65 \times 10^{14}$ & $100 \%$ & $6.9 \mathrm{ppm}$ & $7.99 \times 10^{12}$ & $100 \%$ & $8.8 \times 10^{17}$ \\
\hline
\end{tabular}

$\mathrm{O}_{2}$ and $\mathrm{CO}_{2}$ concentrations remain constant and well mixed for all stellar types.

The planetary surface temperatures range between 297 and $304 \mathrm{~K}$ and Bond albedos range between 0.108 and 0.06 for $\mathrm{M} 0$ to M9 stars, respectively (see Figure 5). These albedos are lower than Earth's Bond albedo of 0.3 around the Sun because the stellar SEDs peak at longer wavelengths for cooler stars, where Rayleigh scattering is less efficient, assuming the same total insolation.

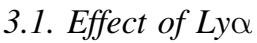

Ly $\alpha$ is the brightest line in the UV spectra for cool stars and accounts for a significant portion of the overall UV flux. However, the intrinsic Ly $\alpha$ flux must be reconstructed to account for interstellar absorption by neutral hydrogen (see Wood et al. 2005; Linsky et al. 2013). For the observed MUSCLES stars, the Ly $\alpha$ line flux is $13 \%-33 \%$ the total UV flux excluding Ly $\alpha$. GJ 1214 has no observed Ly $\alpha$ and only an upper limit that is $0.5 \%$ of the total UV flux (France et al. 2013). In our active models, Ly $\alpha$ ranges from $2 \%$ to $17 \%$ of the total UV flux based on observations of AD Leo. In the inactive models (i.e., no chromosphere) Ly $\alpha$ is negligible compared with total UV flux.
We ran two sensitivity tests of the photochemistry of Earthlike planets to the amount of Ly $\alpha$ flux from their host star. First, we increased the Ly $\alpha$ by a factor of $10^{3}, 10^{6}, 10^{9}, 10^{12}$, and $10^{15}$ above the M0 inactive model, corresponding to flux levels of $2.7 \times 10^{-8}, 2.7 \times 10^{-5}, 2.7 \times 10^{-2}, 2.7 \times 10^{1}$, $2.7 \times 10^{4} \mathrm{ergs} \mathrm{cm}^{-2} \mathrm{~s}^{-1}$, respectively. The highest Ly $\alpha$ value considered is $84 \times$ higher than that of the M0 active model and $180 \times$ higher than the highest observed $\operatorname{Ly} \alpha$ flux in the MUSCLES stellar sample. As seen in Figure 6 (top), increasing the Ly $\alpha$ flux has only a small effect on the photochemistry until the most extreme case considered, where it primarily photolyzes $\mathrm{N}_{2} \mathrm{O}$ and $\mathrm{CH}_{4}$. We run our models to around $60 \mathrm{~km}$, corresponding to a pressure of $1 \times 10^{-4}$ bars. The effect of Ly $\alpha$ will be more pronounced at pressures lower than this, as Miguel et al. (2015) has shown for mini-Neptune atmospheres.

GJ 1214 is the only MUSCLES star to not have a directly detected Ly $\alpha$ flux. An upper limit was placed at $2.4 \times 10^{-15}$ ergs $\mathrm{cm}^{-2} \mathrm{~s}^{-1}$. We artificially set the Ly $\alpha$ flux to be $10^{-2}, 10^{-1}$, $10^{1}, 10^{2}$, and $10^{3}$ times this upper limit. We tested values both above and below the upper limit to see how sensitive an Earthlike planet atmosphere is to changes in $\mathrm{Ly} \alpha$ flux when the rest of the NUV radiation field is still much higher than the inactive model in the previous sensitivity test. As shown in Figure 6 (bottom), the largest changes are seen in the $\mathrm{CH}_{4}$ and to a 
Table 4

Integrated UV Fluxes at the Top of the Atmosphere

\begin{tabular}{|c|c|c|c|c|c|}
\hline $\begin{array}{l}\text { Stellar } \\
\text { Type }\end{array}$ & $\begin{array}{c}\text { Ly } \alpha \text { TOA } \\
1210-1222 \AA \\
\left(\operatorname{ergs~} \mathrm{cm}^{-2} \mathrm{~s}^{-1}\right)\end{array}$ & $\begin{array}{c}\text { FUV minus Ly } \alpha \\
\text { TOA 1222-2000 } \\
\left(\text { ergs cm }{ }^{-2} \mathrm{~s}^{-1}\right)\end{array}$ & $\begin{array}{c}\text { FUV TOA } \\
1000-2000 \AA \\
\left(\operatorname{ergs~} \mathrm{cm}^{-2} \mathrm{~s}^{-1}\right)\end{array}$ & $\begin{array}{c}\text { NUV TOA } \\
2000-3200 \AA \\
\left(\text { ergs } \mathrm{cm}^{-2} \mathrm{~s}^{-1}\right)\end{array}$ & $\begin{array}{c}\text { Full UV TOA } \\
1000-3200 \AA \\
\left(\operatorname{ergs~cm}^{-2} \mathrm{~s}^{-1}\right)\end{array}$ \\
\hline \multicolumn{6}{|c|}{ Active } \\
\hline M0 A & $6.9 \times 10^{2}$ & $2.4 \times 10^{2}$ & $9.4 \times 10^{2}$ & $1.4 \times 10^{3}$ & $2.3 \times 10^{3}$ \\
\hline M1 A & $5.6 \times 10^{2}$ & $2.0 \times 10^{2}$ & $7.6 \times 10^{2}$ & $1.1 \times 10^{3}$ & $1.9 \times 10^{3}$ \\
\hline M2 A & $4.4 \times 10^{2}$ & $1.6 \times 10^{2}$ & $6.1 \times 10^{2}$ & $8.5 \times 10^{2}$ & $1.5 \times 10^{3}$ \\
\hline M3 A & $3.5 \times 10^{2}$ & $1.2 \times 10^{2}$ & $4.7 \times 10^{2}$ & $6.5 \times 10^{2}$ & $1.1 \times 10^{3}$ \\
\hline M4 A & $3.1 \times 10^{2}$ & $1.1 \times 10^{2}$ & $4.2 \times 10^{2}$ & $5.6 \times 10^{2}$ & $9.8 \times 10^{2}$ \\
\hline M5 A & $1.8 \times 10^{2}$ & $6.3 \times 10^{1}$ & $2.5 \times 10^{2}$ & $3.3 \times 10^{2}$ & $5.8 \times 10^{2}$ \\
\hline M6 A & $6.4 \times 10^{1}$ & $2.3 \times 10^{1}$ & $8.8 \times 10^{1}$ & $1.2 \times 10^{2}$ & $2.1 \times 10^{2}$ \\
\hline M7 A & $2.6 \times 10^{1}$ & $9.2 \times 10^{0}$ & $3.6 \times 10^{1}$ & $4.8 \times 10^{2}$ & $8.4 \times 10^{2}$ \\
\hline M8 A & $2.6 \times 10^{1}$ & $9.0 \times 10^{0}$ & $3.5 \times 10^{1}$ & $4.7 \times 10^{2}$ & $8.2 \times 10^{2}$ \\
\hline M9 A & $2.2 \times 10^{1}$ & $7.6 \times 10^{0}$ & $3.0 \times 10^{1}$ & $3.9 \times 10^{2}$ & $6.9 \times 10^{2}$ \\
\hline \multicolumn{6}{|c|}{ Inactive } \\
\hline M0 I & $2.7 \times 10^{-11}$ & $9.4 \times 10^{-3}$ & $9.5 \times 10^{-3}$ & $3.0 \times 10^{2}$ & $3.0 \times 10^{2}$ \\
\hline M1 I & $5.8 \times 10^{-12}$ & $2.9 \times 10^{-3}$ & $2.9 \times 10^{-3}$ & $2.6 \times 10^{2}$ & $2.6 \times 10^{2}$ \\
\hline M2 I & $1.5 \times 10^{-12}$ & $9.1 \times 10^{-4}$ & $9.1 \times 10^{-4}$ & $1.7 \times 10^{2}$ & $1.7 \times 10^{2}$ \\
\hline M3 I & $3.5 \times 10^{-14}$ & $6.9 \times 10^{-5}$ & $6.9 \times 10^{-5}$ & $8.9 \times 10^{1}$ & $8.9 \times 10^{1}$ \\
\hline M4 I & $6.1 \times 10^{-15}$ & $2.2 \times 10^{-5}$ & $2.2 \times 10^{-5}$ & $5.9 \times 10^{1}$ & $5.9 \times 10^{1}$ \\
\hline M5 I & $2.9 \times 10^{-19}$ & $1.5 \times 10^{-8}$ & $1.5 \times 10^{-8}$ & $1.3 \times 10^{1}$ & $1.3 \times 10^{1}$ \\
\hline M6 I & $4.2 \times 10^{-21}$ & $2.6 \times 10^{-9}$ & $2.6 \times 10^{-9}$ & $3.0 \times 10^{0}$ & $3.0 \times 10^{0}$ \\
\hline M7 I & $1.1 \times 10^{-21}$ & $2.6 \times 10^{-10}$ & $2.6 \times 10^{-10}$ & $1.3 \times 10^{0}$ & $1.3 \times 10^{0}$ \\
\hline M8 I & $8.3 \times 10^{-24}$ & $1.7 \times 10^{-10}$ & $1.7 \times 10^{-10}$ & $5.0 \times 10^{-1}$ & $5.0 \times 10^{-1}$ \\
\hline M9 I & $1.8 \times 10^{-24}$ & $1.6 \times 10^{-10}$ & $1.6 \times 10^{-10}$ & $1.3 \times 10^{-1}$ & $1.3 \times 10^{-1}$ \\
\hline \multicolumn{6}{|c|}{ MUSCLES } \\
\hline GJ 832 & $1.5 \times 10^{2}$ & $3.0 \times 10^{0}$ & $1.5 \times 10^{2}$ & $3.5 \times 10^{2}$ & $5.0 \times 10^{2}$ \\
\hline GJ 667C & $1.2 \times 10^{2}$ & $2.4 \times 10^{0}$ & $1.3 \times 10^{2}$ & $2.3 \times 10^{2}$ & $3.6 \times 10^{2}$ \\
\hline GJ 1214 & $1.4 \times 10^{0}$ & $9.9 \times 10^{1}$ & $1.1 \times 10^{2}$ & $1.8 \times 10^{2}$ & $2.8 \times 10^{2}$ \\
\hline GJ 436 & $6.1 \times 10^{1}$ & $7.1 \times 10^{0}$ & $6.8 \times 10^{1}$ & $2.3 \times 10^{2}$ & $2.9 \times 10^{2}$ \\
\hline GJ 581 & $4.2 \times 10^{1}$ & $6.8 \times 10^{0}$ & $5.0 \times 10^{1}$ & $2.7 \times 10^{2}$ & $3.2 \times 10^{2}$ \\
\hline GJ 876 & $3.4 \times 10^{1}$ & $1.1 \times 10^{1}$ & $4.9 \times 10^{1}$ & $1.1 \times 10^{2}$ & $1.6 \times 10^{2}$ \\
\hline
\end{tabular}

smaller extent in stratospheric $\mathrm{H}_{2} \mathrm{O}$ concentrations. $\mathrm{O}_{3}$ and $\mathrm{N}_{2} \mathrm{O}$ remain relatively constant through the five order of magnitude change in Ly $\alpha$ flux.

Figures 4 and 6 show that it is important to characterize the entire UV spectrum, including the NUV and the base level flux between emission lines, to understand future observations of extrasolar planet atmospheres. Ly $\alpha$ is one of the most important lines to characterize.

\section{RESULTS: SPECTRA OF EARTH-LIKE PLANETS ORBITING M DWARFS}

Spectra of Earth-like planets orbiting M dwarfs with varying UV activity levels show measureable differences in spectral feature depths. In the visible, the depth of absorption features is primarily sensitive to the abundance of the species, while in the IR, both the abundance and the temperature difference between the emitting/absorbing layer and the continuum influence the depth of features.

We assume full phase (secondary eclipse) for all spectra presented to show the maximum flux that can be observed. Figures 7-12 show an Earth-size planet to determine the specific flux and planet-to-star contrast ratio. A super-Earth with up to twice Earth's radius will provide 4 times more flux and a better contrast ratio. No noise has been added to these model planetary spectra to provide inputs for a wide variety of instrument simulators for both secondary eclipse and direct detection missions.

\subsection{Earth-like Visible/NIR Spectra (0.4-4 $\mu \mathrm{m})$}

Figure 7 shows the reflected visible and NIR spectra from 0.4 to $2 \mu \mathrm{m}$ of Earth-like planets around the M dwarf grid of active, inactive, and MUSCLES stars, using the SED of the host star. We assume Earth-analog cloud cover. The highresolution spectra calculated with $0.1 \mathrm{~cm}^{-1}$ steps have been smoothed to a resolving power of 800 using a triangular smoothing kernel to show the individual features more clearly. The Earth-Sun spectrum is shown for comparison as a dashed black line.

Owing to the increased stellar flux at shorter wavelengths for a G-type star, Rayleigh scattering is much more pronounced for FGK stars, which greatly increases the flux from 0.4 to $0.8 \mu \mathrm{m}$ for an Earth-like planet around a hotter star. Since $M$ dwarfs have stronger NIR emission, the 1-2 $\mu \mathrm{m}$ flux is larger around Earth-like planets orbiting $M$ dwarfs than for the Earth-Sun equivalent. The most notable features in the visible/NIR spectra are $\mathrm{O}_{3}$ at $0.6 \mu \mathrm{m}$ (the Chappuis band), $\mathrm{O}_{2}$ at $0.76 \mu \mathrm{m}, \mathrm{H}_{2} \mathrm{O}$ at $0.95 \mu \mathrm{m}$, and $\mathrm{CH}_{4}$ at $0.6,0.7,0.8,0.9,1.0$, and $1.7 \mu \mathrm{m}$. Note that shallow spectral features like the visible $\mathrm{O}_{3}$ feature would require a very high signal-to-noise ratio $(\mathrm{S} / \mathrm{N})$ to be detected. 

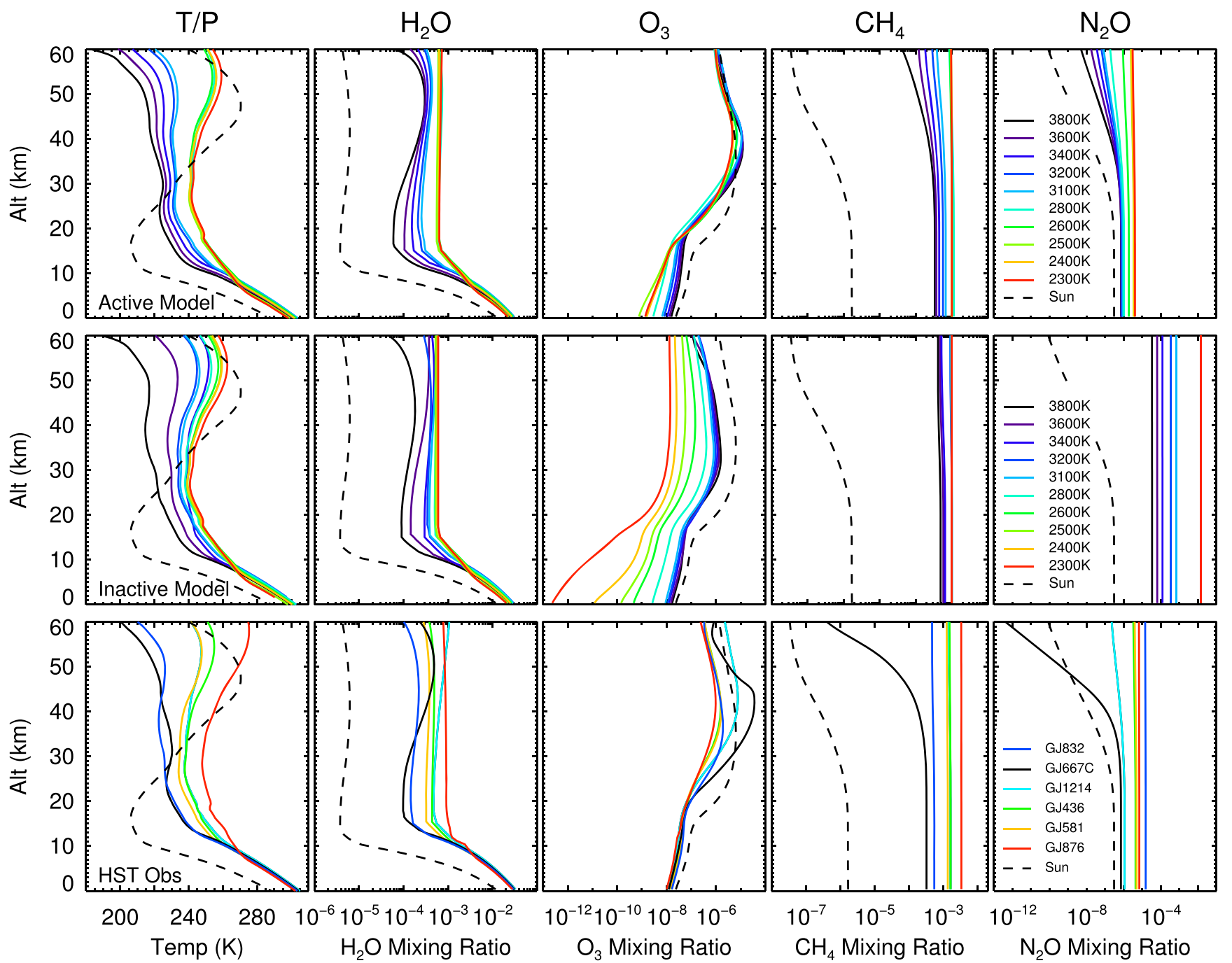

Figure 4. Planetary temperature vs. altitude profiles and mixing ratio profiles profiles for $\mathrm{H}_{2} \mathrm{O}, \mathrm{O}_{3}, \mathrm{CH}_{4}$, and $\mathrm{N}_{2} \mathrm{O}$ (left to right) for an Earth-like planet orbiting the grid of active stellar models (top), inactive stellar models (middle), and MUSCLES stars (bottom). Earth-Sun profiles are shown for comparison (dashed black lines).

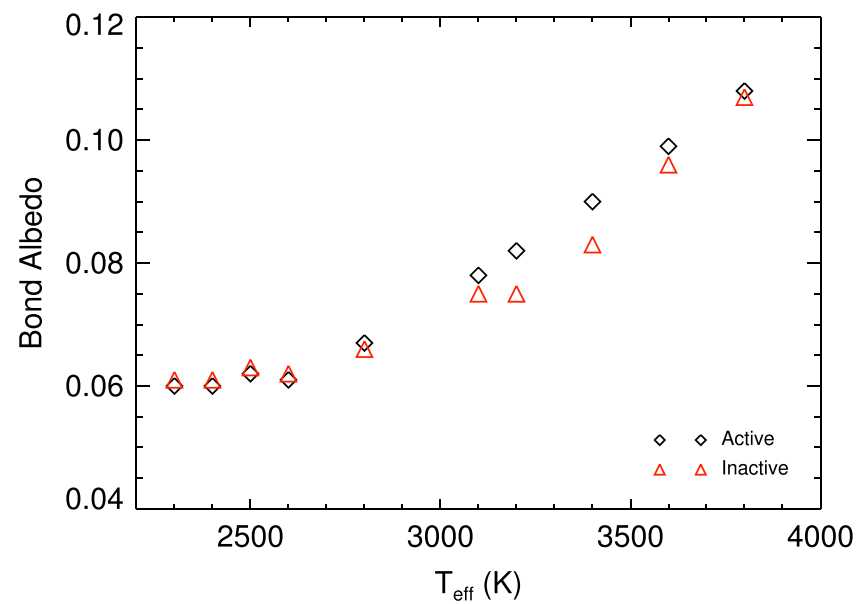

Figure 5. Calculated Bond albedos for Earth-like planets orbiting the M0-M9 active (black diamond) and inactive (red triangle) stellar models.

Figure 8 shows details for one of the most notable feature in the visible, the $\mathrm{O}_{2}$ A band at $0.76 \mu \mathrm{m}$ in both the relative flux as planet-to-star contrast ratio (left) and the reflected emergent flux for a $60 \%$ cloud cover model for M0-M9 model stars (top middle) and for the six MUSCLES stars (top right). Note that the detectability of the $\mathrm{O}_{2}$ feature in reflected light is similar for active and inactive models since the stellar flux at $0.76 \mu \mathrm{m}$ is activity independent. The relative flux shows an equivalently deep feature for each case owing to a constant mixing ratio of $21 \%$. However, the oxygen feature in absolute flux (Figure 8, upper middle panel) becomes increasingly difficult to detect for the later stellar types. For the latest $\mathrm{M}$ stellar types modeled here, the detection of the $\mathrm{O}_{2}$ feature requires a very high $\mathrm{S} / \mathrm{N}$ to detect even if the planet has an active photosynthetic biosphere like Earth (see Figure 7). If one assumes a blackbody radiation Planck function, rather than a realistic stellar model, when calculating the shape of the reflected light curve, the reduction in the feature's depth on moving to later stellar types (i.e., going from blue to red lines in Figure 8, second and third columns) is less pronounced. The $\mathrm{O}_{2}$ feature is pronounced for all MUSCLES stars since none of those stars have a low enough $T_{\text {eff }}$ for the $\mathrm{O}_{2}$ feature to be diminished by the SED. GJ 876 is the coolest MUSCLES star, with $T_{\text {eff }}=3129 \mathrm{~K}$, and in our models the $\mathrm{O}_{2}$ feature becomes most obscured for stars with $T_{\text {eff }}=2300-2600 \mathrm{~K}$. 


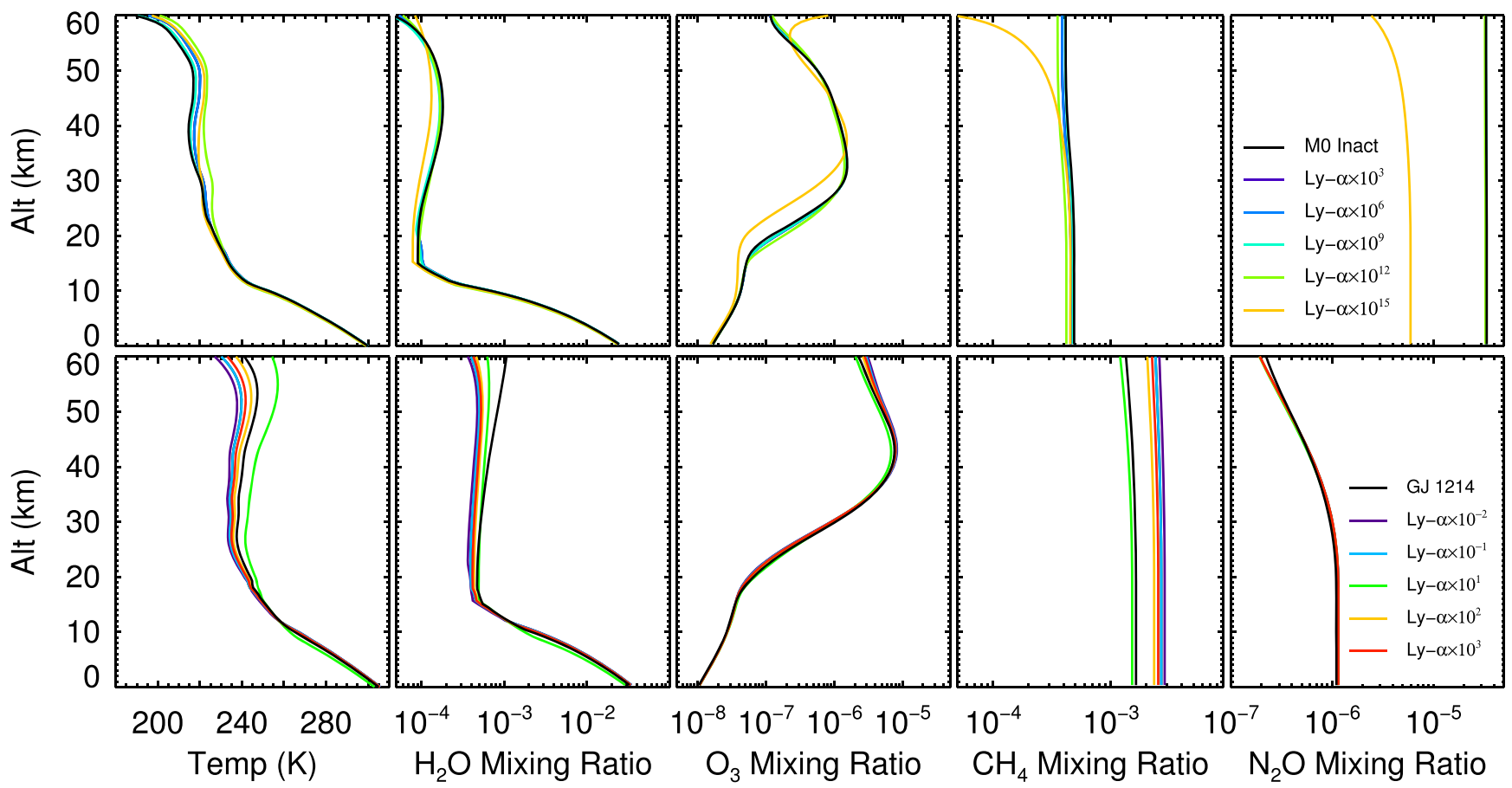

Figure 6. Temperature and $\mathrm{H}_{2} \mathrm{O}, \mathrm{O}_{3}, \mathrm{CH}_{4}$, and $\mathrm{N}_{2} \mathrm{O}$ mixing ratio profiles where the $\mathrm{Ly} \alpha$ has been increased by a factor of $10^{3}, 10^{6}, 10^{9}, 10^{12}$, and $10^{15}$ above the inactive M0 model star, corresponding to flux levels of $2.7 \times 10^{-8}, 2.7 \times 10^{-5}, 2.7 \times 10^{-2}, 2.7 \times 10^{1}$, and $2.7 \times 10^{4} \mathrm{ergs} \mathrm{cm}^{-2} \mathrm{~s}^{-1}$, respectively (top), and for GJ 1214 , where the Ly $\alpha$ upper limit $\left(2.4 \times 10^{-15} \mathrm{ergs} \mathrm{cm}^{-2} \mathrm{~s}^{-1}\right)$ has been multiplied by a factor of $10^{-2}, 10^{-1}, 10^{1}, 10^{2}$, and $10^{3}$ (bottom).

$\mathrm{CH}_{4}$ also has several features of interest in the visible/NIR range at $0.6,0.7,0.8,0.9,1.0$, and $1.7 \mu \mathrm{m}$ (see Figure 9). The $\mathrm{CH}_{4}$ feature is deeper for less active and cooler $\mathrm{M}$ dwarfs. In particular, the $\mathrm{CH}_{4}$ features at $0.8,0.9$, and $1.0 \mu \mathrm{m}$ become much more pronounced for the cooler $\mathrm{M}$ dwarfs and for some of the MUSCLES stars, such as GJ 876.

$\mathrm{H}_{2} \mathrm{O}$ has several features in the visible/NIR at $0.95,1.14$, $1.41,1.86 \mu \mathrm{m}$. These features are deeper for cooler and less active stars.

Clouds increase reflectivity but can also decrease the depth of all observable features compared to the clear sky case. It will be difficult, therefore, to remotely determine the absolute abundance of a molecule without a well-characterized temperature versus pressure distribution and cloud profile.

\subsection{Earth-like Infrared Spectra, IR (4-20 $\mu \mathrm{m})$}

Figure 10 shows the thermal emission spectra from 4 to $20 \mu \mathrm{m}$ of Earth-like planets with Earth-analog cloud cover around the M dwarf grid of active, inactive, and MUSCLES stars using the stellar SED. The high-resolution spectra have been smoothed to a resolving power of 150 using a triangular smoothing kernel to show the resolution expected by JWST. The Earth-Sun IR spectrum is shown for comparison as a dashed black line. Figure 11 shows the individual component gas contributions of the dominant gases $\left(\mathrm{H}_{2} \mathrm{O}, \mathrm{CH}_{4}, \mathrm{CO}_{2}\right.$, $\mathrm{CH}_{3} \mathrm{Cl}, \mathrm{O}_{3}$, and $\mathrm{N}_{2} \mathrm{O}$ ) to the final IR planetary spectrum for an M9 active and inactive stellar model.

The depth of the $\mathrm{O}_{3}$ feature at $9.6 \mu \mathrm{m}$ decreases for planets orbiting cooler and less active $\mathrm{M}$ dwarfs, as expected owing to lower $\mathrm{O}_{3}$ abundances for lower UV incident flux and also to the decreased temperature difference between the $\mathrm{O}_{3}$-emitting layer (around $40 \mathrm{~km}$ ) and the surface temperature, which is larger for the earlier M dwarfs, as seen in Figure 4.
The $\mathrm{CH}_{4}$ feature at $7.7 \mu \mathrm{m}$ decreases in depth for planets orbiting cooler $\mathrm{M}$ dwarfs, despite increasing $\mathrm{CH}_{4}$ abundances making it difficult to remotely determine the $\mathrm{CH}_{4}$ abundance without a well-characterized temperature versus pressure profile. Note that the $7.7 \mu \mathrm{m}$ feature is partially obscured by the wings of the $\mathrm{H}_{2} \mathrm{O}$ feature at $5-8 \mu \mathrm{m}$.

The $\mathrm{N}_{2} \mathrm{O}$ features are the most striking addition in the inactive models with low UV flux. $\mathrm{N}_{2} \mathrm{O}$ has features at 7.75 (overlapping with the $\mathrm{CH}_{4}$ feature), 8.5, 10.65, and $16.9 \mu \mathrm{m}$ (see Figure 11) that become deeper with decreasing UV flux. In the active M0-M9 stellar models we do not see any strong $\mathrm{N}_{2} \mathrm{O}$ features, although it does contribute to the depth of the 7.7 $\mathrm{CH}_{4}$ feature. For the inactive M5-M9 models we see a strong $\mathrm{N}_{2} \mathrm{O}$ feature at $10.65 \mu \mathrm{m}$ and wide absorption from $14-19 \mu \mathrm{m}$ owing to the strong buildup of $\mathrm{N}_{2} \mathrm{O}$ in low UV environments assuming an Earth-like biological surface flux of $7.99 \times 10^{12} \mathrm{~g} \mathrm{yr}^{-1}$ (see Sections 2 and 3). However, even for modest mixing ratios of $15-34 \mathrm{ppm}$ in GJ 832 and the M0 inactive models, respectively, we see a strong $\mathrm{N}_{2} \mathrm{O}$ feature at $16.9 \mu \mathrm{m}$. These concentrations are not much higher than the model mixing ratios calculated for the other MUSCLES stars and the later active $\mathrm{M}$ dwarf models. $\mathrm{N}_{2} \mathrm{O}$ has been considered to be a strong biosignature (see, e.g., Segura et al. 2005; Seager et al. 2012) and will be easier to detect around M dwarfsespecially inactive ones-than around FGK stars.

The $\mathrm{CO}_{2}$ absorption feature at $15 \mu \mathrm{m}$ does not have a central emission peak, usually seen in F and G stars, owing to the more isothermal stratospheres of planets around all $\mathrm{M}$ dwarfs.

The depth of the $\mathrm{H}_{2} \mathrm{O}$ feature at 5-8 $\mu \mathrm{m}$ decreases for later $\mathrm{M}$ dwarfs despite increasing stratospheric $\mathrm{H}_{2} \mathrm{O}$ concentrations.

Clouds reduce the continuum level and the depth of all of the observable features compared to the clear sky case. Therefore, it will be difficult to remotely determine absolute abundances 


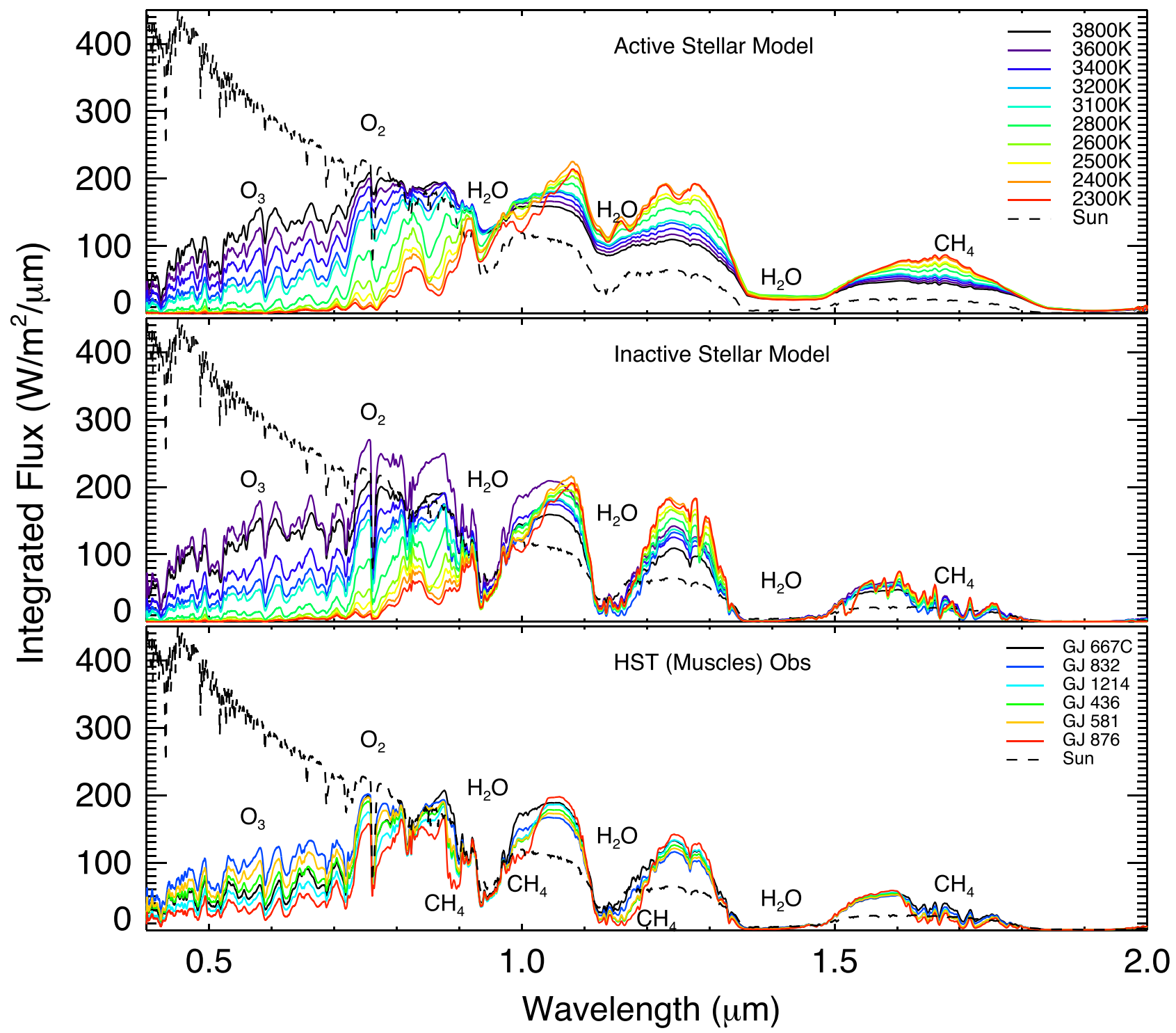

Figure 7. Smoothed, disk-integrated visible/NIR spectra at the TOA for an Earth-like planet for the grid of active stellar models (top), inactive stellar models (middle), and MUSCLES stars (bottom) assuming 60\% Earth-analog cloud coverage model (region 2-4 $\mu \mathrm{m}$ has low integrated flux levels and therefore is not shown here). The Earth-Sun spectrum is shown for comparison as a dashed black line.

without a well-characterized temperature versus pressure distribution and cloud profile.

Observability of Biosignatures: detecting the combination of $\mathrm{O}_{2}$ or $\mathrm{O}_{3}$ and a reducing gas like $\mathrm{CH}_{4}$ in emergent spectra and in secondary eclipse measurements requires either observations in the IR between 7 and $10 \mu \mathrm{m}$ that include the $7.7 \mu \mathrm{m} \mathrm{CH}$ and $9.6 \mu \mathrm{m} \mathrm{O}_{3}$ features, or observations in the visible to NIR between 0.7 and $3 \mu \mathrm{m}$ that include the $0.76 \mu \mathrm{m} \mathrm{O} \mathrm{O}_{2}$ and $2.4 \mu \mathrm{m}$ $\mathrm{CH}_{4}$ features in the observed spectral range. The strengths of absorption features (see Figures 7 and 11 for visible/NIR and IR, respectively) depend on the effective temperature of the host star and its UV flux and vary significantly between stellar types (see Figures 7-11).

In the IR, $\mathrm{CH}_{4}$ at $7.7 \mu \mathrm{m}$ is more easily detected at low resolution for earlier grid stars (M0-M4) than for later grid stars (M5-M9) in the active models.

The $9.6 \mu \mathrm{m} \mathrm{O}_{3}$ feature is deepest in earlier and/or more active M dwarfs, where there are more UV photons. For M5M9 inactive models, $\mathrm{O}_{3}$ is not detectable in low resolution at
$9.6 \mu \mathrm{m}$ owing to the extremely low UV flux for these models. The narrow $\mathrm{O}_{2}$ feature in the visible at $0.72 \mu \mathrm{m}$ becomes weaker for cooler $\mathrm{M}$ dwarfs even though the mixing ratio of $\mathrm{O}_{2}$ remains fixed in our simulations at $21 \%$. This is a consequence of the faint SEDs at shorter wavelengths for both active and inactive late $\mathrm{M}$ stellar models.

For the modern Earth, $\mathrm{N}_{2} \mathrm{O}$ and $\mathrm{CH}_{3} \mathrm{Cl}$ do not contribute substantially to the spectrum owing to their low mixing ratios and will likely be undetectable by the first low-resolution and photon-limited exoplanet atmosphere characterization missions (Selsis 2000; Kaltenegger et al. 2007). However, both $\mathrm{N}_{2} \mathrm{O}$ and $\mathrm{CH}_{3} \mathrm{Cl}$ reach detectable levels in the IR spectra for our models of cooler and less active stars owing to low photolysis rates, even though $\mathrm{N}_{2} \mathrm{O}$ has many absorption features in the IR (see Figure 11).

$\mathrm{N}_{2} \mathrm{O}$ is considered a strong biosignature because there are no significant abiotic sources (Des Marais et al. 2002). One MUSCLES star, GJ 832, has a noticeable $\mathrm{N}_{2} \mathrm{O}$ feature at $17 \mu \mathrm{m}$, with $15 \mathrm{ppm}$ for a modern Earth-like flux. This case is 

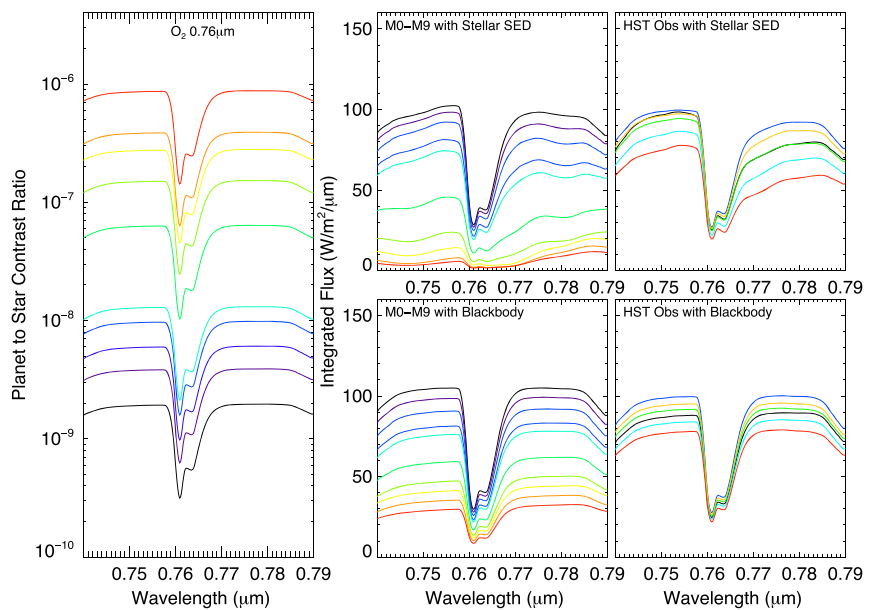

Figure 8. $\mathrm{O}_{2}$ feature at $0.76 \mu \mathrm{m}$ relative flux as planet-to-star contrast ratio (left), and the reflected emergent flux for a $60 \%$ cloud cover model for M0-M9 model stars (top middle) and for MUSCLES stars (top right). Bottom row shows the same but with a blackbody used to calculate the absolute flux. The observable $\mathrm{O}_{2}$ feature becomes increasingly difficult to detect for later stellar types owing to the decreasing stellar flux in this wavelength region. Coloring is the same as in Figure 7.

interesting because GJ 832 has the highest total FUV flux at wavelengths where $\mathrm{N}_{2} \mathrm{O}$ is photolyzed (1000-2400 $\AA$ ). We find that the high Ly $\alpha$ flux of GJ 832 promotes the destruction of $\mathrm{O}_{3}$, producing more $\mathrm{O}\left({ }^{1} D\right)$, which then reacts with $\mathrm{N}_{2}$ to form $\mathrm{N}_{2} \mathrm{O}$.

For planets orbiting inactive M5-M9 star models we observe a sharp increase in $\mathrm{N}_{2} \mathrm{O}$ concentrations as a consequence of few available UV photons. $\mathrm{N}_{2} \mathrm{O}$ features dominate the spectrum for these late, inactive $M$ dwarfs and can be detected in the IR at 4-5 $\mu \mathrm{m}, 8-11 \mu \mathrm{m}$, and $16-19 \mu \mathrm{m}$ (see Figure 11). In an atmosphere dominated by $\mathrm{N}_{2} \mathrm{O}$, such strong absorption throughout the IR could obscure the $7.7 \mu \mathrm{m} \mathrm{CH}_{4}$ feature. It is unknown whether a strong buildup of biotic $\mathrm{N}_{2} \mathrm{O}$ would be physically possible around stars with little or no chromospheric flux. Given the existence of a few GALEX stars with little excess chromospheric NUV flux (Shkolnik \& Barman 2014) and only an upper limit established for the Ly $\alpha$ flux from GJ 1214, a small fraction of $M$ dwarfs could exhibit low UV fluxes. However, the MUSCLES data set shows that all six observed $\mathrm{M}$ dwarfs have sufficient UV flux to photolyze $\mathrm{N}_{2} \mathrm{O}$ and to prevent "runaway" $\mathrm{N}_{2} \mathrm{O}$ buildup.

$\mathrm{CH}_{3} \mathrm{Cl}$ contributes to our IR spectrum from 13 to $14 \mu \mathrm{m}$ in the short-wavelength wing of the $\mathrm{CO}_{2}$ and $\mathrm{N}_{2} \mathrm{O}$ features and could also be detectable, depending on the $\mathrm{CO}_{2}$ and $\mathrm{N}_{2} \mathrm{O}$ concentrations present (see Figure 11).

For clear sky models, the vegetation red edge (VRE) surface feature is detectable in low-resolution spectra owing to the order-of-magnitude-increased VRE reflectance between 0.7 and $0.75 \mu \mathrm{m}$ for all $\mathrm{M}$ grid stars assuming that the exoplanets of these host stars have similar plant life (see Rugheimer et al. 2013 for FGK stars). Clouds partly obscure this feature compared to the clear sky case, although the increase in flux can be seen at $0.7 \mu \mathrm{m}$ in Figure 7 , which includes Earth-like clouds. Owing to the shift in available photons to longer wavelengths for $\mathrm{M}$ dwarfs, a different photosynthesis biochemistry could have evolved, resulting in a different but potentially observable vegetation signature (Kiang et al. 2007).

Observability of Spectral Features: note that we have not added noise to these model spectra in order to be useful as
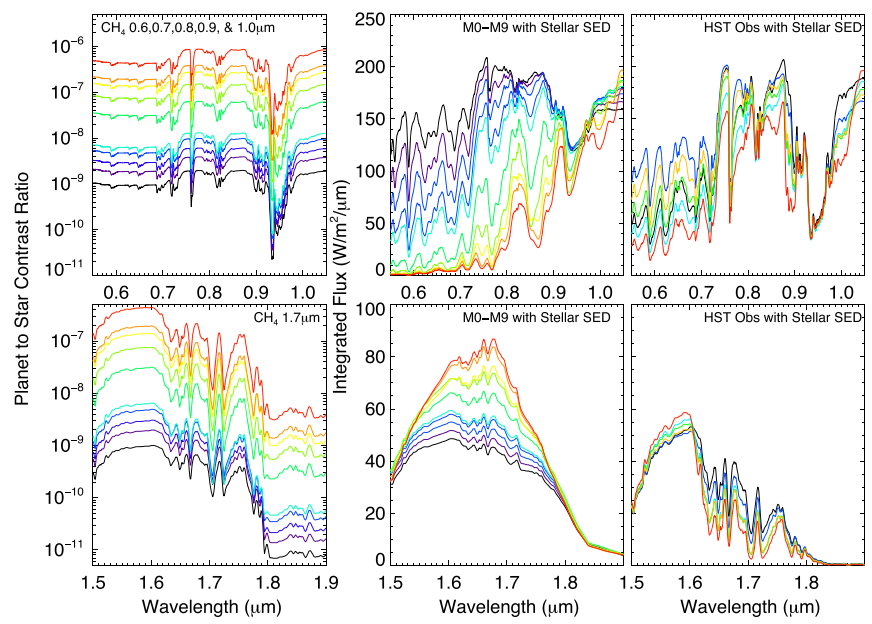

Figure 9. $\mathrm{CH}_{4}$ features at $0.6,0.7,0.8,0.9$, and $1.0 \mu \mathrm{m}$ (top row) and the $\mathrm{CH}_{4}$ feature at $1.7 \mu \mathrm{m}$ (bottom row) for the relative flux as planet-to-star contrast ratio (left) and the reflected emergent flux for a $60 \%$ cloud cover model for M0-M9 model stars (middle) and for MUSCLES stars (right). The observable $\mathrm{CH}_{4}$ feature becomes deeper for planets orbiting cooler and less active star models. Coloring is the same as in Figure 7.

input models for a wide variety of instrument simulators for both secondary eclipse and direct detection simulations. Different instrument simulators for JWST (see, e.g., Deming et al. 2009; Kaltenegger \& Traub 2009) explore the capability of JWST's MIRI and NIRspec instruments to characterize extrasolar Earth-like planets for nearby and luminous host stars. Several groups are providing realistic instrument simulators that can be used to determine the detectability of these absorption features. Future ground- and space-based telescopes are being designed to characterize exoplanets as small as Earth-like planets and will provide opportunities to observe atmospheric features, especially for super-Earths with radii up to twice Earth's radius and therefore four times the flux and planet-to-star contrast ratio levels for Earth-size planets, as shown in Figure 12.

In addition to measuring the size of the planet, future observations will occur at different phases throughout the planet's orbit. The maximum observable planetary flux in the visible spectrum scales with the illuminated fraction of the planet that is visible to the observer. At quadrature, representing an average viewing geometry, the contrast ratios presented in Figure 12 will be a factor of $\sim 2$ lower in the visible. In the IR, the maximum flux remains constant throughout the planet's orbit, assuming a similar temperature on the planet's dayside and nightside.

\section{DISCUSSION}

Our active and inactive stellar models represent the extreme ends of stellar activity for M dwarfs. AD Leo, our active star proxy with $\mathrm{H} \alpha$ in emission, was chosen to represent a young, active star. Our inactive stellar models assume no chromospheric activity, which is the lowest level possible of UV flux. More observations are needed to determine the lowest possible level of UV flux around an M dwarf, which still might be significantly higher than the photosphere-only models. Many M dwarfs exhibit a wide range of activity. Therefore, we use the MUSCLES star database to represent observed M dwarfs with a range of activity between the two extremes. The simulated planets orbiting the MUSCLES stars share more 


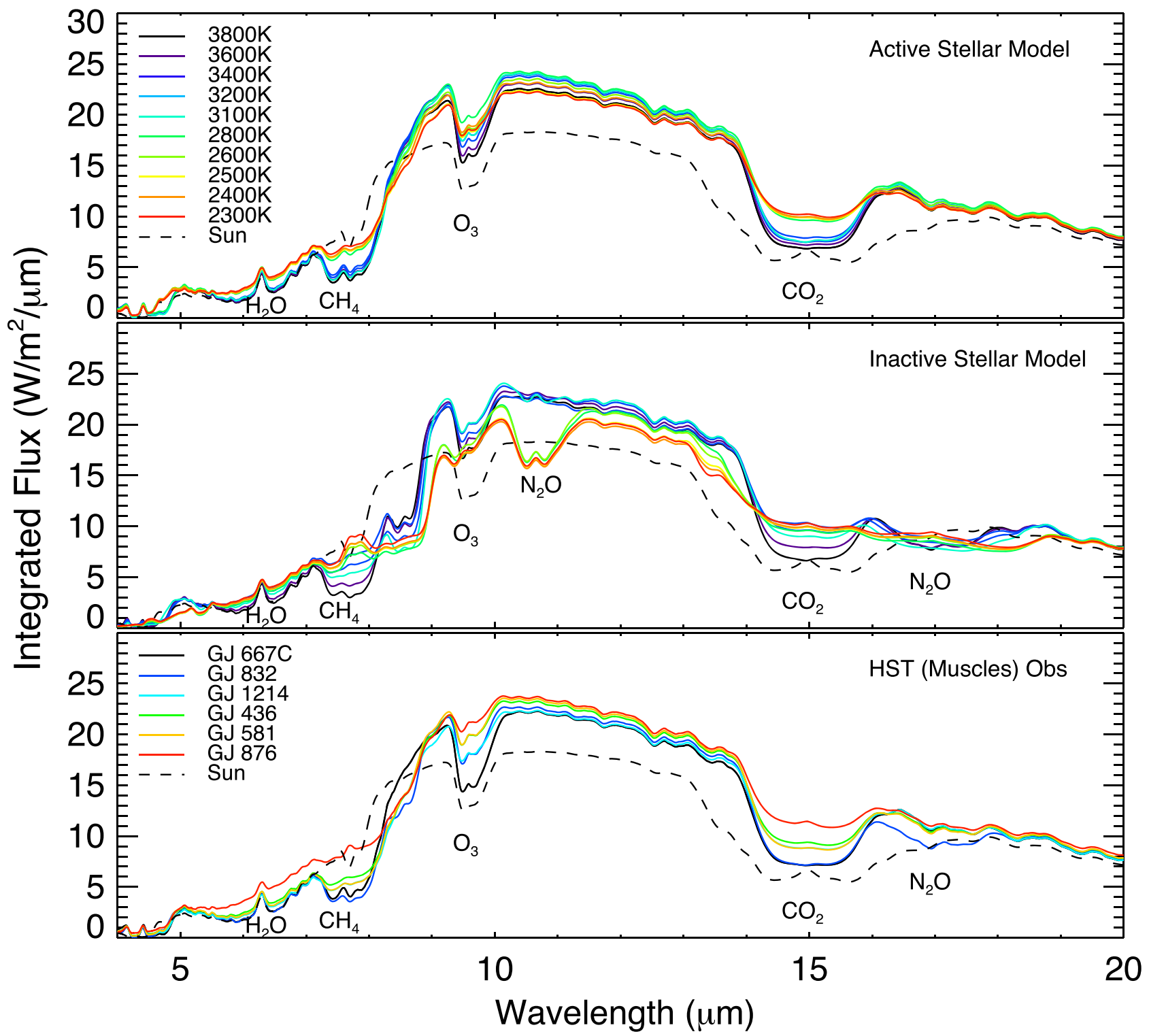

Figure 10. Smoothed, disk-integrated IR spectra at the TOA emitted by an Earth-like planet orbiting the grid of active stellar models (top), inactive stellar models (middle), and MUSCLES stars (bottom) assuming 60\% Earth-analog cloud coverage model. The Earth-Sun spectrum is shown for comparison as a dashed black line.

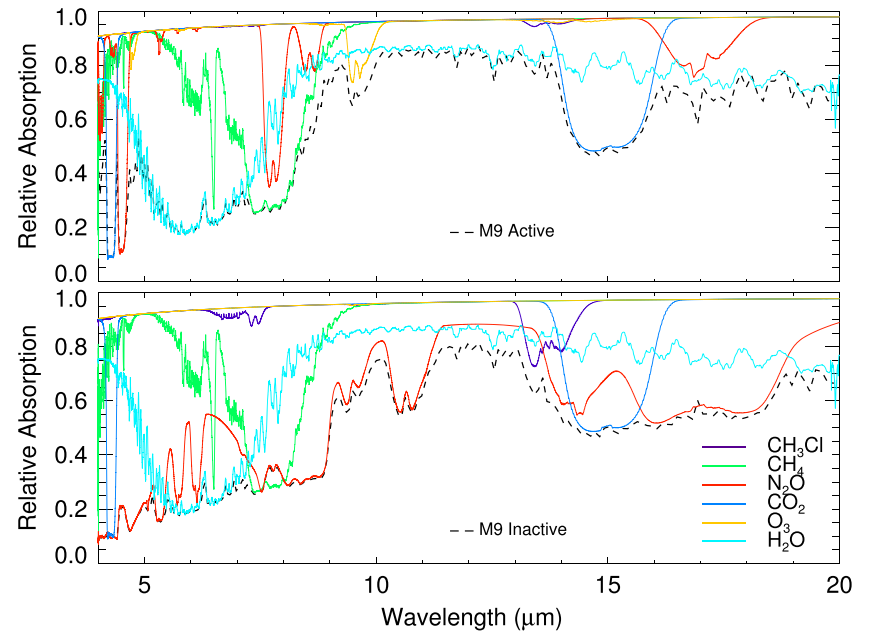

Figure 11. Individual spectral components for $\mathrm{H}_{2} \mathrm{O}, \mathrm{CH}_{4}, \mathrm{CO}_{2}, \mathrm{CH}_{3} \mathrm{Cl}, \mathrm{O}_{3}$, and $\mathrm{N}_{2} \mathrm{O}$ composing the full IR spectra (shown by a dashed black line) for an Earth-like planet orbiting an M9 active stellar model (top) and M9 inactive stellar model (bottom). similarities with the planets orbiting the active stellar models, particularly in terms of $\mathrm{O}_{3}, \mathrm{CH}_{4}$, and $\mathrm{N}_{2} \mathrm{O}$ concentrations, which is to be expected since they have UV fluxes detectable with $H S T$.

Ly $\alpha$ is an important line to characterize because it is the strongest line in the FUV part of an M dwarf spectrum. We note that in addition to properly reconstructing the $\operatorname{Ly} \alpha$ line, determining the base level of flux and other emission lines in the FUV and NUV is also important. This is because Ly $\alpha$ by itself does not significantly impact the photochemistry of an Earth-like planet atmosphere below about $60 \mathrm{~km}$ unless the Ly $\alpha$ flux exceeds the highest observed levels observed for the MUSCLES stars (see Section 3 and Figure 6).

Our present models assume a steady state. Young M dwarfs exhibit strong flares that may impact the atmosphere on timescales relevant to the photochemistry. Future work will consider activity-induced variability in an exoplanet's atmosphere. See Segura et al. (2010) for models on time-dependent behavior of biosignatures for flaring $\mathrm{M}$ dwarfs. 

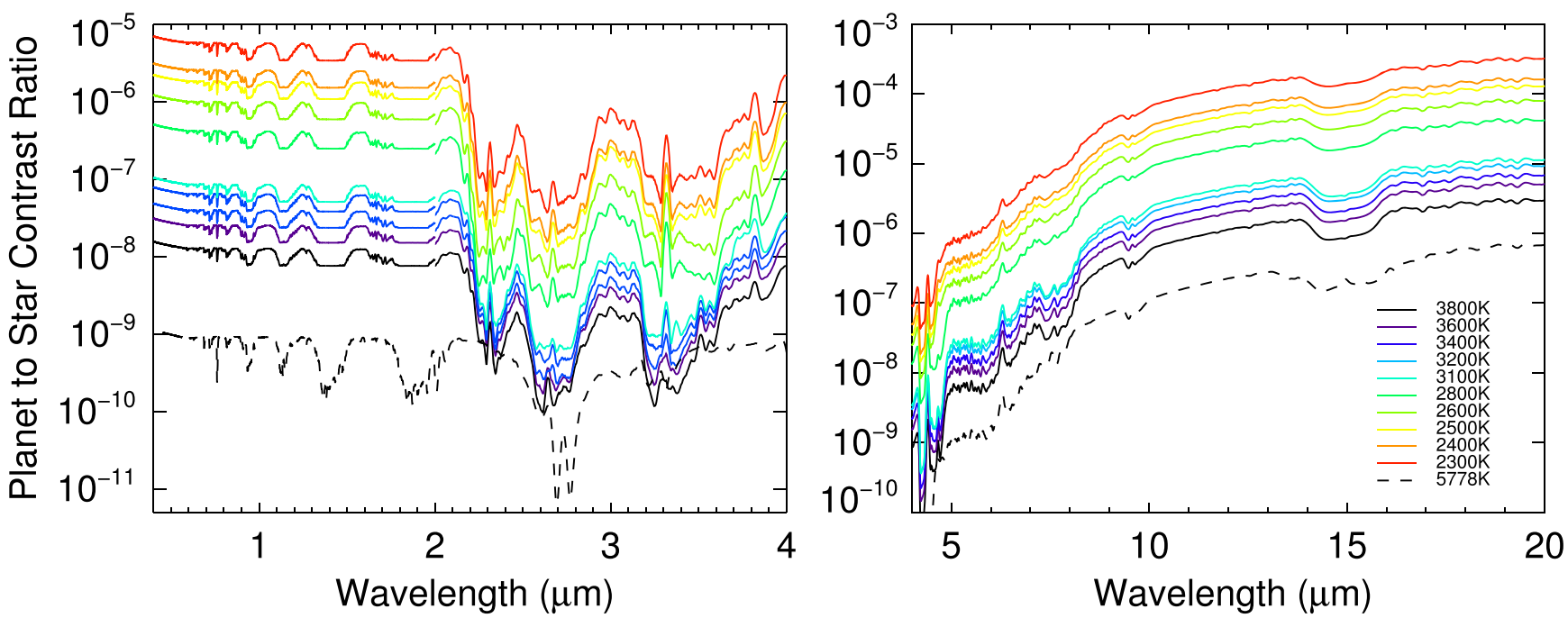

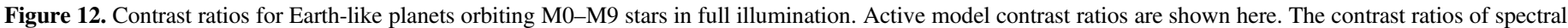

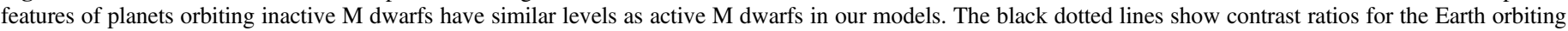
the Sun.

\section{CONCLUSIONS}

We show spectral features for terrestrial atmosphere models of Earth-like planets from the visible to the IR for planets orbiting a grid of M0-M9 host stars $\left(T_{\text {eff }}=2300-3800 \mathrm{~K}\right)$ using active stellar models, inactive stellar models, and the sample of M dwarfs with observed UV fluxes. Our grid comprehensively covers the full $\mathrm{M}$ stellar range and activity from a proxy of an active flare star, AD Leo, to the lower limit of activity with no chromospheric contribution to the UV.

We discuss the atmospheric model results in Section 3 and the detectable spectral features with a focus on how the UV flux environment affects the emergent spectra in Section 4. Increased UV flux environments in M dwarfs are primarily a property of younger and earlier-type stars. Higher UV environments produce increased concentration of $\mathrm{O}_{3}$ from photolysis of $\mathrm{O}_{2}$, increased $\mathrm{O}\left({ }^{1} D\right)$ from photolysis of $\mathrm{O}_{3}$, increased $\mathrm{OH}$ from reaction of $\mathrm{O}\left({ }^{1} D\right)$ and $\mathrm{H}_{2} \mathrm{O}$, decreased stratospheric $\mathrm{H}_{2} \mathrm{O}$, and decreased $\mathrm{CH}_{4}, \mathrm{CH}_{3} \mathrm{Cl}$, and $\mathrm{N}_{2} \mathrm{O}$ from photolysis and reactions with $\mathrm{OH}$ (see Figure 4). Very inactive M dwarfs may have extremely low UV flux levels as suggested by GALEX observations in the NUV. More observations are needed to determine the lower limit of fluxes in the FUV, in particular the Ly $\alpha$ emission. Such low levels of UV flux could lead to a buildup of molecules such as $\mathrm{CH}_{4}$ and $\mathrm{N}_{2} \mathrm{O}$ in the atmosphere of an Earth-like planet, with $\mathrm{N}_{2} \mathrm{O}$ being a strong biosignature owing to a lack of photochemical sources.

Terrestrial planets orbiting early $\mathrm{M}$ spectral type stars are the best targets for observing biosignatures, such as $\mathrm{O}_{2}$ or $\mathrm{O}_{3}$ in combination with a reducing species like $\mathrm{CH}_{4}$. Note that the $\mathrm{O}_{2}$ line becomes increasingly difficult to detect for late $\mathrm{M}$ dwarfs (see Figure 8). While not observable for an exoplanet around an FGK star, $\mathrm{N}_{2} \mathrm{O}$ may be observable at $10.7 \mu \mathrm{m}$ or at $17 \mu \mathrm{m}$ at Earth-level emissions for planets orbiting $M$ dwarfs (see inactive models and model for GJ 832).

Our results provide a grid of atmospheric compositions and model spectra from the visible to the IR for JWST and other future space- and ground-based direct imaging and secondary eclipse missions in terms of instrument design and will help to optimize their observation strategy. The model spectra in this paper are available at www.cfa.harvard.edu/ srugheimer/ Mspectra/.

We especially thank Andrew West and Evgenya L. Shkolnik for useful conversations about $\mathrm{M}$ dwarfs. We also would like to thank Kevin France for discussions concerning the MUSCLES database. This work has made use of the MUSCLES M dwarf UV radiation field database. We would also like to acknowledge support from DFG funding ENP KA 3142/1-1 and the Simons Foundation (290357, Kaltenegger).

The UV radiation field for AD Leo was obtained from the Multimission Archive at the Space Telescope Science Institute (MAST). STScI is operated by the Association of Universities for Research in Astronomy, Inc., under NASA contract NAS526555. Support for MAST for non- $H S T$ data is provided by the NASA Office of Space Science via grant NAG5-7584 and by other grants and contracts.

\section{APPENDIX}

GJ 667C: GJ 667C is an M3-4 dwarf at $6.8 \mathrm{pc}$ (van Leeuwen 2007) with an age estimate greater than $2 \mathrm{Gyr}$ (Anglada-Escudé et al. 2013b). GJ 667C has $T_{\text {eff }}=3350 \mathrm{~K}$, $[\mathrm{Fe} / \mathrm{H}]=-0.55$, and $M=0.330 M_{\odot} \pm 0.019$ (Anglada-Escudé et al. 2013b). Previous estimates had the effective temperature $350 \mathrm{~K}$ higher and the spectral designation to be M1.5 owing to assuming a higher metallicity (see Geballe et al. 2002; Anglada-Escudé et al. 2013b). We use a radius of $R=0.35 R_{\odot}$, consistent with the luminosity $L=0.01370 L \odot$ (Anglada-Escudé \& Tuomi 2012). We merged a PHOENIX BT-Settl spectrum for $T_{\text {eff }}=3350 \mathrm{~K},[\mathrm{Fe} / \mathrm{H}]=-0.55$, $\log (g)=5$, and $v$ sin $i=3 \mathrm{~km} \mathrm{~s}^{-1}$ with the MUSCLES UV spectrum at $2800 \AA$. GJ $667 \mathrm{C}$ has less wavelength coverage than the other MUSCLES stars. We approximated the rest of the UV radiation field from the FUV and NUV SED of GJ 832, which has a similar spectral type (France et al. 2013), scaled to the distance and $\mathrm{Ly} \alpha$ and $\mathrm{Mg}$ II emission line strength of GJ 667C.

GJ 832: GJ 832 is an $\mathrm{M}$ dwarf at $4.95 \mathrm{pc}$ (van Leeuwen 2007) with no age estimate and is the least well characterized star in the MUSCLES program sample. It 
has $T_{\text {eff }}=3620 \mathrm{~K}$ (NStED value interpolated as described in Bessell 1995), $R=0.48 R_{\odot}$ (Johnson \& Wright 1983), $[\mathrm{Fe} / \mathrm{H}]=-0.12$ (Johnson \& Apps 2009), and $\log g=4.7$ (Bailey et al. 2008). We merged a PHOENIX BT-Settl spectrum with $T_{\text {eff }}=3620 \mathrm{~K},[\mathrm{Fe} / \mathrm{H}]=-0.12, \log g=4.7$, and $v \sin i=3 \mathrm{~km} \mathrm{~s}^{-1}$ with the $H S T$ UV spectra from the MUSLES program at $2800 \AA$.

GJ 1214: GJ 1214 is an M6 dwarf at $14.55 \pm 0.13 \mathrm{pc}$ (Anglada-Escudé et al. 2013a) with an age estimate of $6 \pm 3 \mathrm{Gyr}$ (Charbonneau et al. 2009). A more accurate parallax measurement increased the previous distance estimate by $10 \%$, and thus the luminosity and mass have been shifted from previous values as well (Anglada-Escudé et al. 2013a). With the new parallax and luminosity, GJ 1214 has a radius of $0.211 R_{\odot}$, $T_{\text {eff }}=3250 \mathrm{~K}, M=0.176 M_{\odot}$ (Anglada-Escudé et al. 2013a), and $[\mathrm{Fe} / \mathrm{H}]=+0.05$ (Neves et al. 2012; Anglada-Escudé et al. 2013a). We used an upper limit of $v \sin i=1 \mathrm{~km} \mathrm{~s}^{-1}$ (Delfosse et al. 1998; Reiners \& Basri 2008; West \& Basri 2009; Browning et al. 2010) and $\log g=4.991$ (Charbonneau et al. 2009). We merged a PHOENIX BT-Settl spectrum with $T_{\text {eff }}=3250 \mathrm{~K},[\mathrm{Fe} / \mathrm{H}]=+0.05, \log g=4.991$, and $v \sin i=$ $1 \mathrm{~km} \mathrm{~s}^{-1}$ with the MUSCLES UV spectrum at $2800 \AA$ A. GJ 1214 is the only MUSCLES star to have a nondetection of $\operatorname{Ly} \alpha$, and thus an upper limit is used and discussed in depth in France et al. (2013).

GJ 436: GJ 436 is an M3 dwarf at $10.1 \mathrm{pc}$ (van Leeuwen 2007) with an age estimate of 6.5-9.9 Gyr (Saffe et al. 2005). GJ 436 has $T_{\text {eff }}=3416 \mathrm{~K}, R=0.455 \pm 0.018 R_{\odot}$, and $M=0.507(+0.071 /-0.062) M_{\odot}$ (von Braun et al. 2012). GJ 436 has solar metallicity, $[\mathrm{Fe} / \mathrm{H}]=0$ (Rojas-Ayala et al. 2010), $\log g=5.0$ (Maness et al. 2007), and a $v \sin i<1 \mathrm{~km} \mathrm{~s}^{-1}$ (Marcy \& Chen 1992). We merged a PHOENIX BT-Settl spectrum with $T_{\text {eff }}=3416 \mathrm{~K},[\mathrm{Fe} / \mathrm{H}]=0, \log g=5$, and $v \sin i=1 \mathrm{~km} \mathrm{~s}^{-1}$ with the HST UV spectra from the MUSCLES program at $2800 \AA$.

Gl 581: GJ 581 is an M3 dwarf at $6.2 \pm 0.1 \mathrm{pc}$ (van Leeuwen 2007) with age estimate of $7-11 \mathrm{Gyr}$ (Selsis et al. 2007). GJ 581 has $T_{\text {eff }}=3498.0 \pm 56.0 \mathrm{~K}, R=0.299 \pm$ $0.010 R_{\odot}$, and $\log g=4.96 \pm 0.08$ (von Braun et al. 2011). GJ 581 has a metallicity slightly subsolar, $[\mathrm{Fe} / \mathrm{H}]=-0.02$ (RojasAyala et al. 2010), and an upper limit on $v \sin i \leqslant 2.1 \mathrm{~km} \mathrm{~s}^{-1}$ (Delfosse et al. 1998). We merged a PHOENIX BT-Settl spectrum with $T_{\text {eff }}=3498 \mathrm{~K},[\mathrm{Fe} / \mathrm{H}]=-0.02, \log g=4.96$, and $v \sin i=2.1 \mathrm{~km} \mathrm{~s}^{-1}$ (Correia et al. 2010) with the MUSCLES UV spectrum at $2800 \AA$.

GJ 876: GJ 876 is an $\mathrm{M}$ dwarf at $4.69 \mathrm{pc}$ (van Leeuwen 2007) with an age 0.1-5 Gyr (Correia et al. 2010). GJ 876 has $T_{\text {eff }}=3129 \pm 19 \mathrm{~K}$ and $R=0.3761 \pm 0.0059$ $R_{\odot}$ (von Braun et al. 2014). We merged a PHOENIX BT-Settl spectrum with $T_{\text {eff }}=3129 \mathrm{~K},[\mathrm{Fe} / \mathrm{H}]=+0.19$ (Rojas-Ayala et al. 2012), $\log g=4.89$ (Bean et al. 2006), and $v \sin i=$ $1.38 \mathrm{~km} \mathrm{~s}^{-1}$ (Correia et al. 2010) with the MUSCLES UV spectrum at $2800 \AA$.

\section{REFERENCES}

Allard, F. 2014, Proc. IAU Symp. 299, Exploring the Formation and Evolution of Planetary Systems, ed. M. Booth, B. C. Matthews \& J. R. Graham (Cambridge: Cambridge Univ. Press), 271

Anglada-Escudé, G., Rojas-Ayala, B., Boss, A. P., Weinberger, A. J., \& Lloyd, J. P. 2013a, A\&A, 551, A48

Anglada-Escudé, G., \& Tuomi, M. 2012, A\&A, 548, A58

Anglada-Escudé, G., Tuomi, M., Gerlach, E., et al. 2013b, A\&A, 556, A126
Bailey, J., Butler, R. P., Tinney, C. G., et al. 2008, ApJ, 690, 743 Batalha, N. M., Rowe, J. F., Bryson, S. T., et al. 2013, ApJS, 204, 24 Bean, J. L., Benedict, G. F., \& Endl, M. 2006, ApJL, 653, L65 Beichman, C., Lawson, P., Lay, O., et al. 2006, Proc. SPIE, 6268, 626805 Beichman, C. A., Woolf, N. J., \& Lindensmith, C. A. 1999, The Terrestrial Planet Finder (TPF): A NASA Origins Program to Search for Habitable Planets (Pasadena, CA: JPL Publications)

Bessell, M. S. 1995, in Proc. ESO Workshop, The Bottom of the Main Sequence - and Beyond, ed. C. G. Tinney (Berlin: Springer-Verlag), 123

Borucki, W. J., Agol, E., Fressin, F., et al. 2013, Sci, 340, 587

Borucki, W. J., Koch, D. G., Basri, G., et al. 2011, ApJ, 736, 19

Broecker, W. S. 1982, GeCoA, 46, 1689

Browning, M. K., Basri, G., Marcy, G. W., West, A. A., \& Zhang, J. 2010, AJ, 139,504

Cash, W. 2006, Natur, 442, 51

Charbonneau, D., Berta, Z. K., Irwin, J., et al. 2009, Natur, 462, 891

Correia, A. C. M., Couetdic, J., Laskar, J., et al. 2010, A\&A, 511, A21

Delfosse, X., Forveille, T., Perrier, C., \& Mayor, M. 1998, A\&A, 331, 581

Deming, D., Seager, S., Winn, J., et al. 2009, PASP, 121, 952

Des Marais, D. J., Harwit, M. O., Jucks, K. W., et al. 2002, AsBio, 2, 153

Domagal-Goldman, S. D., Segura, A., Claire, M. W., Robinson, T. D., \& Meadows, V. S. 2014, ApJ, 792, 90

Dressing, C. D., \& Charbonneau, D. 2013, ApJ, 767, 95

Forster, P., Ramaswamy, V., Artaxo, P., et al. 2007, in Climate Change 2007: The Physical Science Basis. Contribution of Working Group I to the Fourth Assessment Report of the Intergovernmental Panel on Climate Change, ed. S. Solomon et al. (Cambridge: Cambridge Univ. Press), ch. 2

France, K., Froning, C. S., Linsky, J. L., et al. 2013, ApJ, 763, 149

Gardner, J. P., Mather, J. C., Clampin, M., et al. 2006, SSRv, 123, 485

Geballe, T. R., Knapp, G. R., Leggett, S. K., et al. 2002, ApJ, 564, 466

Grenfell, J. L., Gebauer, S. V., Paris, P., Godolt, M., \& Rauer, H. 2014, Planet. Space Sci., 98, 66

Guzmán-Marmolejo, A., Segura, A., \& Escobar-Briones, E. 2014, AsBio, 13,550

Haqq-Misra, J. D., Domagal-Goldman, S. D., Kasting, P. J., \& Kasting, J. F. 2008, AsBio, 8, 1127

Hawley, S. L., Gizis, J. E., \& Reid, I. N. 1996, AJ, 112, 2799

Hu, R., Seager, S., \& Bains, W. 2012, ApJ, 761, 166

Jenkins, J. S., Ramsey, L. W., Jones, H. R. A., et al. 2009, ApJ, 704, 975

Johnson, H. M., \& Wright, C. D. 1983, ApJS, 53, 643

Johnson, J. A., \& Apps, K. 2009, ApJ, 699, 933

Jones, D., \& West, A. A. 2015, ApJ, submitted

Kaltenegger, L. 2010, ApJL, 712, L125

Kaltenegger, L., Fridlund, M., \& Karlsson, A. 2006, Ap\&SS, 306, 147

Kaltenegger, L., \& Sasselov, D. 2010, ApJ, 708, 1162

Kaltenegger, L., \& Sasselov, D. 2011, ApJL, 736, L25

Kaltenegger, L., Sasselov, D., \& Rugheimer, S. 2013, ApJL, 775, L47

Kaltenegger, L., \& Traub, W. A. 2009, ApJ, 698, 519

Kaltenegger, L., Traub, W. A., \& Jucks, K. W. 2007, ApJ, 658, 598

Kasting, J. F., \& Ackerman, T. P. 1986, Sci, 234, 1383

Kasting, J. F., Holland, H. D., \& Pinto, J. P. 1985, JGR, 90, 10497

Kasting, J. F., Pollack, J. B., \& Crisp, D. 1984, JAtC, 1, 403

Kharecha, P., Kasting, J. F., \& Siefert, J. 2005, Geobiology, 3, 53

Kiang, N. Y., Segura, A., Tinetti, G., et al. 2007, AsBio, 7, 252

Kitzmann, D., Patzer, A. B. C., von Paris, P., Godolt, M., \& Rauer, H. 2011a, A\&A, 531, A62

Kitzmann, D., Patzer, A. B. C., von Paris, P., Godolt, M., \& Rauer, H. 2011b, A\&A, 534, A63

Linsky, J. L., France, K., \& Ayres, T. 2013, ApJ, 766, 69

Lovelock, J. E. 1975, RSPSB, 189, 167

Maness, H. L., Marcy, G. W., Ford, E. B., et al. 2007, PASP, 119, 90

Marcy, G. W., \& Chen, G. H. 1992, ApJ, 390, 550

Miguel, Y., Kaltenegger, L., Linsky, J. L., \& Rugheimer, S. 2015, MNRAS, 446,345

Neves, V., Bonfils, X., Santos, N. C., et al. 2012, A\&A, 538, A25

Pavlov, A. A., \& Kasting, J. F. 2002, AsBio, 2, 27

Pavlov, A. A., Kasting, J. F., Brown, L. L., Rages, K. A., \& Freedman, R. 2000, JGR, 105, 11981

Quintana, E. V., Barclay, T., Raymond, S. N., et al. 2014, Sci, 344, 277

Rauer, H., Gebauer, S. v., Paris, P., et al. 2011, A\&A, 529

Reiners, A., \& Basri, G. 2008, ApJ, 684, 1390

Ricker, G. R., Winn, J. N., Vanderspek, R., et al. 2014, Proc. SPIE, 9143 914320

Rodler, F., \& López-Morales, M. 2014, ApJ, 781, 54

Rojas-Ayala, B., Covey, K. R., Muirhead, P. S., \& Lloyd, J. P. 2010, ApJL, 720, L113 
Rojas-Ayala, B., Covey, K. R., Muirhead, P. S., \& Lloyd, J. P. 2012, ApJ, 748, 93 Rugheimer, S., Kaltenegger, L., Zsom, A., Segura, A., \& Sasselov, D. 2013, AsBio, 13, 251

Saffe, C., Gómez, M., \& Chavero, C. 2005, A\&A, 443, 609

Sagan, C., Thompson, W. R., Carlson, R., Gurnett, D., \& Hord, C. 1993, Natur, 365,715

Schindler, T. L., \& Kasting, J. F. 2000, Icar, 145, 262

Seager, S., Schrenk, M., \& Bains, W. 2012, AsBio, 12, 61

Segura, A., Kasting, J. F., Meadows, V., et al. 2005, AsBio, 5, 706

Segura, A., Krelove, K., Kasting, J. F., et al. 2003, AsBio, 3, 689

Segura, A., Meadows, V. S., Kasting, J. F., Crisp, D., \& Cohen, M. 2007, A\&A, 472, 665

Segura, A., Walkowicz, L. M., Meadows, V., Kasting, J., \& Hawley, S. 2010, AsBio, 10, 751

Selsis, F. 2000, in ESA Special Publication 451, Darwin and Astronomy: The Infrared Space Interferometer, ed. B. Schürmann (Noordwijk: ESA), 133

Selsis, F., Kasting, J. F., Levrard, B., et al. 2007, A\&A, 476, 1373

Shkolnik, E. L., \& Barman, T. S. 2014, ApJ, in press (arXiv:1407.1344)

Snellen, I. A. G., de Kok, R. J., le Poole, R., Brogi, M., \& Birkby, J. 2013, ApJ, 764,182
Tian, F., France, K., Linsky, J. L., Mauas, P. J. D., \& Vieytes, M. C. 2014, E\&PSL, 385, 22

Toon, O. B., McKay, C. P., Ackerman, T. P., \& Santhanam, K. 1989, JGR, 94, 16287

Traub, W. A., Levine, M., Shaklan, S., et al. 2006, Proc. SPIE, 6268, $62680 \mathrm{~T}$

Traub, W. A., \& Stier, M. T. 1976, ApOpt, 15, 364

Udry, S., Bonfils, X., Delfosse, X., et al. 2007, A\&A, 469, L43

van Leeuwen, F. 2007, A\&A, 474, 653

von Braun, K., Boyajian, T. S., Kane, S. R., et al. 2011, ApJL, 729, L26

von Braun, K., Boyajian, T. S., Kane, S. R., et al. 2012, ApJ, 753, 171

von Braun, K., Boyajian, T. S., van Belle, G. T., et al. 2014, MNRAS, 438, 2413

Walkowicz, L. M., \& Hawley, S. L. 2009, AJ, 137, 3297

West, A. A., \& Basri, G. 2009, ApJ, 693, 1283

West, A. A., Hawley, S. L., Walkowicz, L. M., et al. 2004, AJ, 128, 426

West, A. A., Morgan, D. P., Bochanski, J. J., et al. 2011, AJ, 141, 97

Wood, B. E., Redfield, S., Linsky, J. L., Müller, H.-R., \& Zank, G. P. 2005, ApJS, 159,118

Wordsworth, R., \& Pierrehumbert, R. 2014, ApJL, 785, L20

Wordsworth, R. D., Forget, F., Selsis, F., et al. 2011, ApJL, 733, L48 